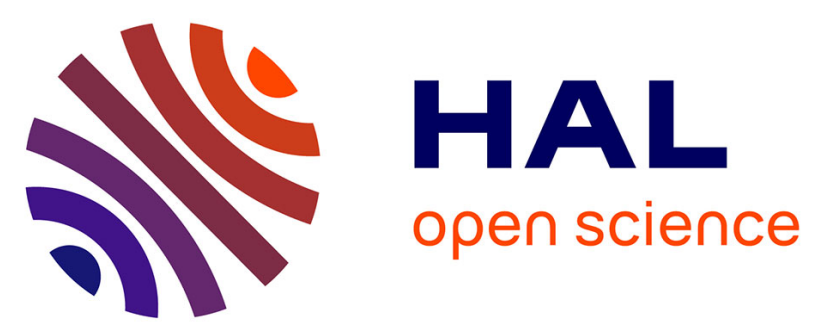

\title{
Microbiota tryptophan metabolism induces aryl hydrocarbon receptor activation and improves alcohol-induced liver injury
}

Laura Wrzosek, Dragos Ciocan, Cindy Hugot, Madeleine Spatz, Margot Dupeux, Camille Houron, Vanessa Liévin-Le Moal, Virginie Puchois, Gladys Ferrere, Nicolas Trainel, et al.

\section{To cite this version:}

Laura Wrzosek, Dragos Ciocan, Cindy Hugot, Madeleine Spatz, Margot Dupeux, et al.. Microbiota tryptophan metabolism induces aryl hydrocarbon receptor activation and improves alcohol-induced liver injury. Gut, 2021, 70 (7), pp.1299-1308. 10.1136/gutjnl-2020-321565 . hal-03329872

\section{HAL Id: hal-03329872 \\ https://hal.sorbonne-universite.fr/hal-03329872}

Submitted on 31 Aug 2021

HAL is a multi-disciplinary open access archive for the deposit and dissemination of scientific research documents, whether they are published or not. The documents may come from teaching and research institutions in France or abroad, or from public or private research centers.
L'archive ouverte pluridisciplinaire HAL, est destinée au dépôt et à la diffusion de documents scientifiques de niveau recherche, publiés ou non, émanant des établissements d'enseignement et de recherche français ou étrangers, des laboratoires publics ou privés. 


\section{Microbiota tryptophan metabolism induces aryl hydrocarbon receptor activation and} improves alcohol-induced liver injury

Laura Wrzosek ${ }^{1,2 \$}$, Dragos Ciocan ${ }^{1,2,3 \$}$, Cindy Hugot ${ }^{1,2}$, Madeleine Spatz ${ }^{1,2}$, Margot Dupeux ${ }^{1,2,4}$, Camille Houron ${ }^{1,2}$, Vanessa Liévin-Le Moal ${ }^{1,2}$, Virginie Puchois ${ }^{1,2}$, Gladys Ferrere ${ }^{1,2}$, Nicolas Trainel $^{1,2}$, Françoise Mercier-Nomé ${ }^{5}$, Sylvère Durand ${ }^{6}$, Guido Kroemer ${ }^{7}$, Cosmin Sebastian Voican $^{1,2,3}$, Patrick Emond ${ }^{8,9}$, Marjolene Straube ${ }^{10}$, Harry Sokol ${ }^{10,11,12}$, Gabriel Perlemuter ${ }^{1,2,3 *}$, and Anne-Marie Cassard ${ }^{1,2^{*}}$

\section{(1)}

${ }^{\$}$ These authors contributed equally to the work.

*Corresponding authors:

Anne-Marie Cassard : INSERM U996, 32 rue des carnets 92190 Clamart, France.

$$
\text { Tel: }+33141288037
$$

Fax: +33146327993

cassard.doulcier@u-psud.fr

Gabriel Perlemuter : INSERM U996, 32 rue des carnets, 92190 Clamart, France.

Tel: +33141288037

Fax: +33146327993

gabriel.perlemuter@aphp.fr.

${ }^{1}$ Université Paris-Saclay, Inserm U996, Inflammation, Microbiome and Immunosurveillance, 92140, Clamart, France.

${ }^{2}$ Institut Paris-Sud d'Innovation Thérapeutique (IPSIT), IFR141, Faculté de Pharmacie, Univ Paris-Sud, Université Paris-Saclay, Châtenay-Malabry, France. ${ }^{3}$ AP-HP, HepatoGastroenterology and Nutrition, Hôpital Antoine-Béclère, Clamart, France. ${ }^{4}$ AP-HP, Anatomie-Pathologique, Hôpital Kremlin-Bicêtre, le Kremlin-Bicêtre, France. ${ }^{5}$ Université Paris-Saclay, Inserm, CNRS, Institut Paris Saclay d'Innovation thérapeutique, 92296, Châtenay-Malabry, France. ${ }^{6}$ Metabolomics and Cell Biology Platforms, Gustave Roussy Cancer Campus, Villejuif, France. ${ }^{7}$ Gustave Roussy Cancer Campus, Villejuif, France; INSERM, U1138, Paris, France; Equipe 11 labellisée par la Ligue Nationale contre le Cancer, Centre de Recherche des Cordeliers, Paris, France; Université Paris Descartes/Paris V, Sorbonne Paris Cité, Paris, France; Metabolomics and Cell Biology Platforms, Gustave Roussy Cancer Campus, Villejuif, France; Université Pierre et Marie Curie, Paris, France; Pôle de Biologie, Hôpital Européen Georges Pompidou, AP-HP, Paris, France; Karolinska Institute, 
1 Department of Women's and Children's Health, Karolinska University Hospital, Stockholm, 2 Sweden. ${ }^{8}$ UMR 1253, iBrain, Université de Tours, Inserm, Tours, France. ${ }^{9}$ CHRU de Tours, 3 Service de Médecine Nucléaire In Vitro, Tours, France. ${ }^{10}$ Sorbonne Université, Inserm, Centre 4 de Recherche Saint-Antoine, CRSA, AP-HP, Hôpital Saint Antoine, Service de 5 Gastroenterologie, F-75012 Paris, France. ${ }^{11}$ Gastroenterology Department, Saint-Antoine 6 Hospital, Assitance Publique-Hôpitaux de Paris (AP-HP), Paris, France. ${ }^{12}$ INRA, UMR1319 7 Micalis, AgroParisTech, Jouy-en-Josas, France.

8

$9 \quad$ Electronic word count: $\mathbf{4 0 5 2}$

\section{Number of figures and tables: 5 figures}

13

14 Keywords: alcoholic hepatitis, indole, fecal microbiota transplantation, human microbiota 15 associated mice, fiber, pectin 


\section{ABSTRACT}

2 Objective: Chronic alcohol consumption is an important cause of liver-related deaths. Specific 3 intestinal microbiota profiles are associated with susceptibility or resistance to alcoholic liver

4 disease in both mice and humans. We aimed to identify the mechanisms by which targeting

5 intestinal microbiota can improve alcohol-induced liver lesions.

6 Design: We used human associated mice, a mouse model of alcoholic liver disease transplanted 7 with the intestinal microbiota of alcoholic patients and used the prebiotic, pectin, to modulate 8 the intestinal microbiota. Based on metabolomic analyses, we focused on microbiota tryptophan 9 metabolites, which are ligands of the aryl hydrocarbon receptor (AhR). Involvement of the AhR 10 pathway was assessed using both a pharmacological approach and AhR-deficient mice.

11 Results: Pectin treatment modified the microbiome and metabolome in human microbiotaassociated alcohol-fed mice, leading to a specific fecal signature. High production of bacterial tryptophan metabolites was associated with an improvement of liver injury. The AhR agonist FICZ (6-formylindolo (3,2-b) carbazole) reduced liver lesions, similarly to prebiotic treatment. Conversely, inactivation of the $a h r$ gene in alcohol-fed AhR knock-out mice abrogated the beneficial effects of the prebiotic. Importantly, patients with severe alcoholic hepatitis have low

17 levels of bacterial tryptophan derivatives that are AhR agonists.

18 Conclusions: Improvement of alcoholic liver disease by targeting the intestinal microbiota involves the AhR pathway, which should be considered as a new therapeutic target. 


\section{SUMMARY BOX}

\section{What is already known about this subject?}

3 - The intestinal microbiota is a causal factor of alcohol-induced liver lesions in mice and 4 humans.

5 - Pectin is able to prevent alcohol-induced liver injury in mice by altering the intestinal 6 microbiota.

7 - The protective effect of pectin is associated with an improvement of gut barrier function.

\section{What are the new findings?}

10 - Moderate amount of pectin can cure alcoholic liver disease in the context of the human 11 microbiota.

- Pectin increases the production of tryptophan metabolites, which are aryl hydrocarbon receptor (AhR) ligands, by the microbiota, improving gut barrier function.

- Pharmacological activation of the AhR by FICZ, an exogenous AhR ligand, is sufficient to simulate the effect of pectin.

- AhR deficiency abrogates the beneficial effect of pectin, demonstrating a major role for the

17 AhR pathway in the protective effect of the intestinal microbiota.

\section{How might it impact on clinical practice in the foreseeable future?}

Therapeutic options in alcohol-induced liver injury are limited. Our results show that targeting intestinal microbiota using moderate amount of pectin can reverse alcohol-induced liver injury through the AhR pathway. Modifying intestinal microbiota to increase its production of AhR ligands or AhR ligand administration could be new therapeutic targets for alcoholic patients. 


\section{INTRODUCTION}

Chronic alcohol consumption is a major cause of liver-related deaths [1]. Severe alcoholic hepatitis (sAH) is a life-threading form of alcoholic liver disease (ALD), with few therapeutic options [2]. Recent studies have shown that specific microbiota profiles are associated with susceptibility or resistance to alcohol-induced liver lesions in both mice and humans, opening new therapeutic options $[3,4,5,6]$. Moreover, the production of cytolysin by Enterococcus faecalis has been specifically shown to be involved in ALD development in 30\% of sAH patients and its eradication by phagotherapy improves liver injury in a mouse model of ALD [7, 8]. Aside from deleterious bacteria, it is also relevant to identify bacteria that can protect patients from developing alcohol-induced liver lesions and to understand the molecular mechanisms involved in such protective effects.

Several studies have reported that modulation of intestinal microbiota (IM) composition by fecal microbiota transfer [4, 5,9] or treatment with Akkermansia muciniphila [10], Roseburia intestinalis [11], other probiotics, or fiber/prebiotics [12,13] can improve liver injury in mouse models of ALD. Molecular mechanisms by which microbiota alterations can improve alcoholinduced liver lesions are poorly understood and involve changes in the gut barrier and bacterial metabolites.

Disruption of the gut barrier correlates with endotoxemia and the severity of ALD in humans and mice $[4,5,14]$. This disruption is associated with a decrease in the production of mucus and anti-microbial peptides and the disruption of tight junctions [15, 16, 17]. Modulation of the IM by fecal transfer, prebiotics, or probiotics restores a leaky gut [4, 6, 13, 15]. Moreover, changes in IM composition in patients with ALD induce modifications in microbiota-associated metabolites, including short chain fatty acids (SCFAs) and bile acids [12], and are involved in the severity of alcohol-induced liver injury [5, 18, 19]. Among microbiota-associated metabolites, tryptophan-derived indoles, produced by a large number of bacteria, including Bacteroides, are ligands of the aryl hydrocarbon receptor (AhR). AhR signaling improves the function of the intestinal barrier by increasing local expression of IL-22 [20, 21] and, consequently, increases expression of antimicrobial proteins [22]. Moreover, the abundance of Bacteroidetes and level of plasma tryptophan decrease after acute alcohol administration in humans, suggesting impaired tryptophan metabolism [23].

Here, we aimed to identify the mechanisms by which targeting the IM with a prebiotic can improve alcohol-induced liver lesions. We used human microbiota-associated mice (HMA), which were transplanted with the IM of patients with sAH, to work in the context of the human 
1 microbiota. We used the prebiotic fiber, pectin, to alter the IM. We and others have already

2 shown that pectin can modify the mouse IM and prevent ALD by improving the leaky gut-

3 barrier. However, the molecular mechanisms involved in this process and the effects of pectin

4 in the context of the human IM are still unknown $[4,6]$. We now demonstrate that pectin 5 reshapes the microbiome in the context of the human microbiota and not only prevents but also

6 reverses alcohol-induced liver injury in mice. Metabolomic studies showed that changes in the

7 microbiota composition also induced alterations in bacterial tryptophan metabolism, leading to

8 the high production of indole derivatives, which activate the AhR. Pharmacological treatment

9 of mice with an AhR agonist simulating the effect of pectin on the liver and reversed ALD, 10 whereas inactivation of the ahr gene in knock-out mice abrogated the effects of the beneficial 11 microbiota in alcohol-fed mice. The results observed in the humanized mice are also supported 12 by a decreased level of AhR agonists in patients with sAH, suggesting that AhR may be a new 13 therapeutic target in ALD. 
MATERIAL AND METHODS

Mice. Female C57BL/6J mice (Janvier laboratory, Le Genest, France) were kept in humidity and temperature-controlled rooms, on a 12-hour light-dark cycle. Mice had access to a chow diet and water ad libitum before the study. Body weight and food intake were measured three times a week. All our experimental procedures were validated by the ethical committees and the French veterinary minister (2015052715405651_v2 (APAFIS\#729) and 2017042314557080v1(APAFIS\#4788).

Treatments. In all our experiments, pectin was given at D21, as a curative treatment, using an alternative Lieber DeCarli diet containing different concentrations of pectin from apple $(0.4 \%$, 1\%, 2\% and 6.5\%, w /w, Sigma-Aldrich, Saint Quentin Fallavier, France). To treat mice by an AhR agonist, the 6-formylindolo (3,2-b)carbazole (Ficz; Sigma-Aldrich) was re-suspended in dimethyl sulfoxide (DMSO; Sigma-Aldrich), diluted in olive oil (Sigma-Aldrich) and administered intraperitoneally. Ficz $(1 \mu \mathrm{g} /$ mouse $)$ treatment was injected three times during the last week when mice were exposed to the maximum dose of alcohol (5\%) and until euthanization. Control mice received DMSO vehicle diluted in olive oil intraperitoneally alone for the Ficz treatment group.

Fecal microbiota transfer. Mice received feces from alcoholic patients with severe alcoholic hepatitis as previously described [5, 24]. Two set of independent experiments were performed, a first set with feces from two different patients $\left(F_{1}\right.$ and $\left.F_{2}\right)$ and a second set with feces from one patient $\left(\mathrm{F}_{3}\right)$. Briefly, feces from human patients were recovered and immediately stored at $4^{\circ} \mathrm{C}$ in an anaerobiosis generator (Genbox, Biomérieux, Capronne, France) to favour the preservation of anaerobic bacteria. All samples were processed within $24 \mathrm{~h}$. Feces were rapidly diluted 100-fold in Brain Heart Infusion (BHI, Becton Dickinson) supplemented with $0.5 \mathrm{mg} / \mathrm{ml}$ L-cysteine (Sigma-Aldrich, St-Louis, MO, USA) and 20\% skim milk (Becton Dickinson) (vol/vol) and stored in aliquots at $-80^{\circ} \mathrm{C}$. This ready-to-use fecal suspension was used for FMT to mice.

Mice were fasted $1 \mathrm{~h}$ and then subjected to bowel cleansing by oral-gastric gavage with PEG (polyethylene glycol, Macrogol 4000, Fortrans, Ipsen Pharma, France). Four hours later, mice received the human feces by oral gastric gavage (200 $\mu 1$ of resuspended feces prepared as described above). Mice were then allowed free access to food and water. FMT was repeated twice a week for four weeks. Bowel cleansing was only performed on day 1. 
1 Patients. Two groups of patients were included in the study: patients with severe alcoholic 2 hepatitis (sAH) and alcoholic patients without alcoholic hepatitis and without cirrhosis (noAH). 3 All patients were admitted to the hepato-gastroenterology department of Antoine-Béclère 4 University Hospital, Clamart, France. Alcoholic patients were eligible for inclusion if they had consumed at least $50 \mathrm{~g}$ of alcohol per day over the previous year, were negative for hepatitis $\mathrm{B}$ surface antigens, and seronegative for antibodies against hepatitis $\mathrm{C}$ virus (HCV). Exclusion criteria were gastrointestinal bleeding, bacterial infection, hepatocellular carcinoma or other carcinoma, acute pancreatitis, other severe associated disease, diabetes mellitus, dyslipidemia, presence of anti-HIV antibodies, and antibiotic or probiotic intake in the last 3 months. A standardized questionnaire was used, to collect information about alcohol consumption [25]. Severe alcoholic hepatitis was suspected in patients with a Maddrey score $>32$ and was confirmed by a liver biopsy (histological score for $\mathrm{AH} \geq 6$ with neutrophilic infiltration)[26, 27]. Feces from 3 independent patients with severe alcoholic hepatitis were used for the fecal transfer in mice.

The study was carried out in accordance with the Helsinki Declaration and was approved by the Ile de France VII ethics committee (Bicêtre Hospital, 94270 le Kremlin-Bicêtre, France). All the participants provided written informed consent.

Statistical analyses. Results are represented as the mean \pm SEM. Statistical comparison was performed by first testing the normality of the data using the Shapiro-Wilk test of normality and then performing unpaired Mann-Whitney, unpaired t-test, Kruskal-Wallis or ANOVA tests as appropriate (Graphpad Prism, Graphpad Software Inc, La Jolla, California, USA); p < 0,05 was considered to be statistically significant. ${ }^{*} \mathrm{p}<0.05,{ }^{* *} \mathrm{p}<0.01,{ }^{* * *} \mathrm{p}<0.001$.

See the Supplementary section for the sources of materials and detailed methods.

\section{RESULTS}

\section{Altering the intestinal microbiota reverses alcohol-induced liver lesions}

We tested whether altering the intestinal microbiota can reverse the progression of alcohol-induced liver lesions using human microbiota-associated mice (HMA) [24, 28]. We used HMA mice transplanted with the microbiota from sAH patients, as we have previously shown that the intestinal microbiota worsens alcohol-induced liver lesions in this model [5]. Pectin, a dietary fiber known to favor the growth of specific bacterial genera, such as 
1 Bacteroides [4, 29], which are reduced by alcohol intake [27], was used to alter the intestinal 2 microbiota. Conventional (Alc) and HMA mice from three independent patients with sAH (Alc $3 \mathrm{~F}_{1}$, Alc $\mathrm{F}_{2}$, Alc $\mathrm{F}_{3}$ ) were fed alcohol using the Lieber DeCarli (LDC) diet, as described previously [4] (Fig. 1a). The clinical characteristics of the donors with sAH are presented in Supplementary Table 1. Principal coordinate analysis (PCoA) showed that alcohol, human microbiota transfer, and pectin treatment induced changes in intestinal microbiota composition (unweighted Unifrac, ANOSIM, r =0.59, $\mathrm{P}<0.001$, Fig. 1b and Supplementary Fig. 1a, c). These changes included an increase in the Bacteroides genus in the pectin-treated group (Fig. $1 c$ and Supplementary Fig. 1b, d).

HMA alcohol-fed mice developed liver lesions during the first week of alcohol intake, as shown by liver TG accumulation and an increase in ALT levels and several markers of inflammation (Supplementary Fig. 2 a-e). At this point, mice were treated with pectin in order to alter intestinal microbiota. Pectin did not modify the alcohol absorption (Fig 1a and Supplementary Fig. 2f). Changing microbiota by using pectin, reversed alcohol-induced liver lesions in HMA mice fed alcohol. These mice (Alc $\left.F_{1} P\right)$ had lower levels of ALT (Fig. 1d), liver TG (Fig. 1e), steatosis (Fig. 1f), and liver inflammation markers (ccl2, thf $\alpha$, ill $\beta$ and $c c l 3)$ (Fig. 1g) than Alc $F_{1}$ and Alc mice. We obtained similar modifications of the intestinal microbiota and recovery of alcohol-induced liver lesions in HMA mice using feces from two other independent sAH patients (Alc $\mathrm{F}_{2}$ and Alc $\mathrm{F}_{3}$, Supplementary Fig. 3 and 4).

Altering the intestinal microbiota using high-dose of fiber may be associated with poor tolerance (bloating, abdominal distension) [30]. We therefore tested the efficacy of lower doses of pectin on ALD. Two percent pectin induced similar changes of the alcohol-induced liver lesions and gut barrier function while improving treatment tolerance (Supplementary Fig. 4). Pectin treatment induced dose-dependent changes in the intestinal microbiota (Supplementary Fig. 5a-d). Among the specific changes observed in the LEfSe analysis, Alc F 32 showed an increase in the abundance of the Bacteroidetes phylum, a decrease in the abundance of the Firmicutes phylum, and an increase in the abundance of Bacteroides and Lactobacillus genera, similar to that of Alc $\mathrm{F}_{3}$ P6.5 relative to Alc F 3 (Supplementary Fig. 5d). However, the increase in the abundance of Proteobacteria and Enterobacteriaceae observed in the Alc F 36.5 mice was not observed in the Alc F 32 mice (Supplementary Fig. 5e). 
Disruption of the intestinal barrier correlates with the severity of liver injury in ALD [4,

$25,6,15,31]$. Alcohol-induced gut barrier disruption results in a decrease in the level of the antimicrobial peptides reg3 $\beta$ and reg3y and mucus production $[4,6]$. Restoration of these functions is required to improve alcohol-induced liver injury [4, 6, 16, 32]. Altering the intestinal microbiota improved liver injury through improvement of the gut barrier function, as shown by an increase in antimicrobial peptide ( $\operatorname{reg} 3 \beta$ and reg $3 \gamma$ ) mRNA levels in the colon and ileum, and the proportion of goblet cells (Supplementary Fig. 6a-g). This was associated with an improvement of intestinal permeability, as shown by an increase in tight junction proteins (ZO-1 and occludin) as shown by mRNA levels and immunoflourescence in the colon and ileum (Supplementary Fig. 6h-i and Supplementary Fig. 7) and a decrease of bacterial translocation into the liver (Supplementary Fig. 6j). These results show that altering the microbiota improves the gut barrier and reverses alcohol-induced liver injury, despite on-going heavy alcohol consumption.

\section{Altering the intestinal microbiota modifies its functions and the fecal metabolome}

We next explored the functional impact of altering the intestinal microbiota using pectin by generating the predicted metagenome using Phylogenetic Investigation of Communities by Reconstruction of Unobserved States (PICRUSt) [33]. A total of 4,977 KEGG orthologs were assigned to 146 metabolic pathways and 115 structural complex modules. Pectin-treated mice showed a higher number of bacterial genes involved in carbohydrate, lipid, and amino-acid metabolism (Fig. 2a). Conversely, control mice showed a higher number of bacterial genes involved in amino-acid, energy, and cofactor and vitamin metabolism. We obtained similar predicted metagenome profiles in mice transplanted with the intestinal microbiota of the two other independent patients $\left(\mathrm{F}_{2}\right.$ and $\left.\mathrm{F}_{3}\right)($ Supplementary Table 2).

We further studied whether such changes in the bacterial pathways induce alterations in the fecal metabolome by performing targeted metabolomic profiling. PCA and heatmaps showed that altering the intestinal microbiota induced a specific fecal metabolomic profile (Fig. 2b, c). Enrichment analysis using Metabolom Analyst led to the identification of 52 pathways that were modified between Alc $F_{1}$ and Alc $F_{1} P$ mice (FDR $<0.05$ ) (Fig. 2d and Supplementary Table 3). These pathways belong to the metabolism of amino acids (lysine, tyrosine, tryptophan, valine, leucine, isoleucine, and beta-alanine), carbohydrates (starch and sucrose, pentose and glucose interconversion, and ascorbate) lipids, and vitamins (biotin and ascorbate). Many of the changes in the fecal metabolomic pathways belong to those highlighted 
1 by the predicted bacterial metagenome (Fig. 2a, d). These results were also confirmed when 2 using lower doses of pectin (Supplementary Fig. 8).

Among the amino acids of which the levels were modified by pectin, we specifically identified a decrease in the levels of tryptophan and indole, precursors of microbiota-derived tryptophan metabolites (Fig. 2e). We then performed specific metabolomic profiling of tryptophan metabolites, as we observed an increase in the abundance of Bacteroides, taxa that can metabolize tryptophan into indole derivatives, in pectin-treated mice. We observed a decreased level of indole-3-acrylic acid in alcohol-fed conventional and HMA mice as compared to control mice and a decrease in overall AhR agonists (sum of 3-indoxyl sulfuric acid, 5-Methoxy-3-indoleacetic acid, indole-3-acetic acid, indole-3-acrylic acid, indole-3aldehyde, indole-3-lactic acid, indole-3-propionic acid) in alcohol-fed HMA mice. These changes were restored after pectin treatment with an overall increase in total AhR agonists in pectin treated mice (Fig. 2f).

\section{Activation of the AhR pathway improves alcohol-induced liver injury}

Altering the intestinal microbiota with pectin in the HMA mouse model of ALD reverses alcohol-induced injury and is associated with changes in the microbiota and tryptophan metabolism. We therefore studied the role of the AhR pathway, which can be activated by bacterial tryptophan metabolites. We analyzed the expression of cyplal and its repressor ahrr, target genes of AhR activation in the colon. Their expression in the colon (Fig. 3a), together with that of il22 [20] and ill7 (Fig. 3b), which are also controlled by AhR activation [34], increased after altering the intestinal microbiota by pectin treatment. We then assessed the direct involvement of $\mathrm{AhR}$ in the improvement of alcohol-induced injury by treating mice with an AhR agonist, 6-formylindolo (3,2-b) carbazole (Ficz). Treatment of Alc mice with Ficz increased the expression of AhR target genes Cyplal and Scdl in the liver (Fig. 3c) and decreased alcohol-induced liver lesions, with a decrease in ALT, liver TG, and inflammatory marker levels (Fig. 3d, e). Treatment with Ficz also increased antimicrobial peptide levels in the colon and ileum (Fig. 3f, g) but only the expression of Reg $3 \gamma$ in the ileum reached statistical significance, simulating the effects of pectin. We next tested whether AhR signaling mediates the effects of pectin using alcohol-fed mice deficient for AhR (AhR KO). Although pectin treatment still restored ALT levels in alcohol-fed AhR KO mice, it could neither alleviate steatosis (Fig. 4a, b) nor restore cyplal and ahrr mRNA levels (Fig. 4c). Accordingly, restoration of $i l 22, \operatorname{reg} 3 \beta$, and $r e g 3 \gamma$ mRNA levels by pectin treatment was also abrogated (Fig. 
1 4d). Overall, these data show that the effects of pectin are, at least partially, mediated by AhR 2 pathways.

3

4 Tryptophan metabolism is impaired in patients with severe alcoholic hepatitis

We next explored the relevance of impaired tryptophan metabolism in the context of 6 human disease and analyzed fecal and serum samples of alcoholic patients with (sAH) or 7 without alcoholic hepatitis (noAH) (Supplementary Table 4). There were no differences in the 8 fecal levels of tryptophan, kynurenine, or AhR agonists between alcoholic patients, regardless 9 of the severity of the liver injury (noAH or sAH) (data not shown). There was also no difference in the serum level of kynurenine between alcoholic patients (Fig. 5a). Conversely, serum levels of tryptophan and AhR agonists were lower in sAH patients than in noAH patients (Fig. 5a). We also found negative correlations between serum levels of Trp and AST $(r=-0.6, p<0.01)$, bilirubin $(\mathrm{r}=-0.7, \mathrm{p}<0.001)$, prothrombin time $(\mathrm{r}=-0.7, \mathrm{p}<0.001)$, and MELD score $(\mathrm{r}=-$ 0.6, $\mathrm{p}<0.001)$ (Fig. 5b). This suggest that tryptophan metabolism is impaired in patients with alcoholic hepatitis and that the modulation of AhR could be a new therapeutic target. 


\section{DISCUSSION}

The IM plays a role in the pathophysiology of ALD and bacterial composition contributes to the severity of liver injury, independently of alcohol intake $[2,5]$. Bacteria interact directly with the host and indirectly through a large panel of bacterial metabolites [35]. Impairment of several bacterial metabolic functions has been shown to exacerbate ALD, including that of bacterial synthesis of saturated long-chain fatty acids [36], bile acids [5, 19, 27], and tryptophan [37]. In ALD, disruption of the intestinal barrier correlates with the severity of liver lesions $[4,15,31]$. The role of the IM in the development of a leaky gut is associated with decreased levels of antimicrobial Reg 3 peptides and decreased mucus production $[4,16]$. Altering the IM using probiotics or prebiotics in murine models can prevent ALD by modulating these functions $[6,10,38,39,40]$. Specifically, pectin, a fiber that modulates the intestinal microbiota, can prevent alcohol-induced liver injury by improving gut barrier function [4]. Nevertheless, a preventive effect of such a treatment is not relevant for patients with alcoholuse disorders that have ALD. Therefore, we addressed the effect of pectin in a mouse model of ongoing alcohol administration after the onset of liver injury. We focused on bacterial indole derivatives, as the molecular mechanisms by which fiber-induced changes of the IM improve ALD have not been elucidated.

Here, we show that pectin, used as a curative treatment, is able to reverse alcoholinduced liver injury in the context of the human microbiota. We used HMA transplanted with the feces of alcoholic patients with sAH. Liver injury in these mice is worse than that of wildtype alcohol-fed mice. Improvement of liver lesions is associated with improved gut barrier function, including the restoration of mucus production and antimicrobial Reg 3 peptide levels. Pectin, as a dietary fiber, is known to favor the growth of specific bacterial genera, such as Bacteroides [4, 29]. In alcoholic patients, sAH is associated with a decrease in the abundance of Bacteroidetes and changes in IM function [5, 41]. A similar decrease in the abundance of Bacteroidetes has also been observed in animal models of ALD [4]. Here, we show that pectin induces an increase in the abundance of Bacteroides, regardless of the effective dose.

A high dose of pectin (6.5\%) was also associated with an increased abundance of Proteobacteria, which could pose safety concerns, as several species of this phylum are considered to be opportunistic pathogens [42]. Moreover, fermentable fiber (including pectin) has been reported to induce an increase in the abundance of Proteobacteria and hepatocarcinoma in several animal models (TLR5, TLR4, and Lcn2-deficient mice). This is due to the inability of the innate immunity in the gut to prevent the translocation of Proteobacteria species [43]. However, in our study, the lower dose of pectin (2\%) used to improve intestinal tolerance to a 
1 diet rich in fiber abrogated Proteobacteria overgrowth and achieved the beneficial effects that

2 we observed on alcohol-induced liver lesions with a pectin-enriched diet. The amount of pectin to administrate in patients to match the minimal effective dose described in our study $(2 \%)$ would be of $40 \mathrm{~g} /$ day. Of note, the recommended daily dose of fiber intake ranges between 30 $38 \mathrm{~g} /$ day in men and 21-25 g/day in women [44]. Nevertheless, the amount of fiber consumed by humans is dependent on their diet. It has been suggested that omnivores consumed less than $23 \mathrm{~g}$ of fibers/day, vegetarians significantly more (37 g/day) and vegans the most ( $47 \mathrm{~g} /$ day) [45]. Patients with chronic liver disease (viral and alcoholic cirrhosis) have a lower intake of vegetables which are rich in fibers [46]. Moreover, a high-fiber diet has been related to regression of NAFLD [47] and recent epidemiological data showed that dietary fiber intake, especially soluble fibers (such as pectin), is inversely associated with the risk of several chronic diseases and with mortality [48]. Studies investigating pectin administration in different conditions used up to $60 \mathrm{~g} /$ day and reported good tolerance [49]. The main side effect was bloating but individual sensitivity to develop side effects is highly variable [50]. These data suggest that a moderate amount of pectin may be a promising and safe alimentary complement in the management of alcoholic patients.

We further analyzed intestinal metabolites to investigate the mechanisms by which pectin-induced modifications of the IM reduce alcohol-induced gut and liver injuries. The microbiota reshaped by pectin harbored more genes involved in amino-acid and xenobiotic metabolism. This metagenomic prediction was confirmed by the quantification of fecal metabolites. We specifically identified a decrease in tryptophan levels and an increase in the level of indole derivatives. These metabolites are only produced by the intestinal microbiota from tryptophan [37]. Several microbiota-derived tryptophan metabolites are able to activate the AhR, thus playing a key role in gut homeostasis through the regulation of anti-microbial peptide and mucus production by IL-22 [21]. Moreover, it has been previously shown that IL22 is down-regulated in alcohol-fed mice and oral treatment with recombinant IL-22 or bacteria that produce this cytokine prevents alcohol-induced liver injury [37, 51]. AhR activation has been shown to improve inflammatory bowel disease [52] and metabolic syndrome [37, 53, 54]. Moreover, hepatic AhR activation prevents HSC activation and the expression of genes required for liver fibrogenesis by disrupting the interaction of Smad3 with $\beta$-catenin [55]. Here, we show that the improvement of mucus and antimicrobial peptide production by pectin is associated with the restoration of AhR-responsive gene expression, including that of IL-22, Cyp1a1, and ahrr. Conversely, low levels of $\operatorname{reg} 3 \beta$, reg $3 \gamma$, and mucus production in untreated alcohol-fed mice correlated with lower levels of il22, Cyp1a1, and ahrr. 
We also treated mice with an AhR agonist, FICZ, to address the direct involvement of AhR in the effects of pectin. FICZ treatment was sufficient to mediate a reduction in alcoholinduced injury. In contrast, pectin treatment of AhR-deficient alcohol-fed mice had only a minimal effect on alcohol-induced liver lesions suggesting that the effects of pectin treatment in our model of ALD are not solely medicated by AhR. Indeed, pectin induces broad changes at the microbial and metabolomic level and other mechanisms independent of AhR could also mediate the effect observed in our study.

The relevance of impaired tryptophan metabolism in the context of human disease was confirmed by reduced serum levels of tryptophan and AhR agonists in patients with sAH. Conversely, there were no differences in the fecal levels of tryptophan, kynurenine, or AhR agonists between alcoholic patients, regardless of the severity of the liver injury (noAH or sAH). However, it has been reported that patients with alcoholic hepatitis have lower levels of fecal indole-3-acetic acid and indole-3-lactic acid than healthy patients who do not consume alcohol [37]. These discrepancies suggest that alcohol induces impairment of tryptophan metabolism independently of liver disease.

Our results provide the basis for further studies in patients with ALD that will aim to correct the AhR-ligand deficiency. Indeed, it has been recently shown that Lactobacillus reuteri, which is known to produce AhR agonists, improves ALD [37], as well as treatment with a direct agonist, such as indole-3 acetic acid. Moreover, treatment with Lactobacillus reuteri can also improve metabolic syndrome [53] and colitis [56, 57] in animal models. Indole3-pyruvic acid, an AhR agonist, improves experimental colitis [58] and indigo, a tryptophan metabolite that activates the AhR, is effective in inducing remission in patients with ulcerative colitis [59].

In conclusion, our study shows that alcohol-induced liver lesions can be reversed by modifying AhR-agonist production by the IM. As there is no treatment that can reverse alcoholinduced liver lesions other than liver transplant, modulation of the AhR pathways by supplementation with prebiotics, AhR ligand-producing bacteria, or pharmacological AhR ligands, may hold promise in the development of new therapeutic approaches to ALD. 
1 Acknowledgments. The authors thank Mylène Levant, Baptiste Lecomte, and Sarah Mendez

2 for follow-up of the mice, the Plaimmo Platform, PHIC platform (Morgan Ocimek and Séverine

3 Domenichini), and Nicolas Sorhaindo for plasma quantification (Plateforme de Biochimie,

4 Bichat). We also thank the NED team (Olivier Zemb and Béatrice Gabinaud) and the GeT-

$5 \quad$ PlaGe platform for their help with the sequencing data.

Disclosures: DC received travel funds from Biocodex and Gilead, lecture fees from Gilead, and royalties from John Libbey Eurotext. HS received unrestricted study grants from Danone, Biocodex, and Enterome and board membership, consultancy, or lecture fees from Carenity, Abbvie, Astellas, Danone, Ferring, Mayoly Spindler, MSD, Novartis, Roche, Tillots, Enterome, Maat, BiomX, Biose, Novartis, and Takeda and is a co-founder of Exeliom Biosciences. GP received travel funds from Janssen and Gilead, consulting fees from Bayer, Biocodex, Roche, Gilead, Pierre Fabre, and Servier, and royalties from Elsevier-Masson, Solar, Flammation/Versilio, and John Libbey Eurotext. AMC received travel funds and consulting fees from Biocodex and royalties from Elsevier-Masson, Solar, Flammation/Versilio, and John Libbey Eurotext. All other authors declare no conflicts of interest.

Financial support: This work was supported by INSERM, Université Paris-Sud, "Fondation pour la recherche médicale" (FRM), the National French Society of Gastroenterology (SNFGE), "Association Française pour l'Etude du Foie" (AFEF), "Fondation pour la Recherche en Alcoologie" (FRA/IREB), "Institut de Recherches Internationales Servier" (IRIS), and "Groupement transversal INSERM sur le microbiote" (GPT microbiota). DC received a grant from Biocodex. MSp received a grant from the Laboratory of Excellence LERMIT supported by the "Agence Nationale de la Recherche" (ANR-10-LABX-33). MD received a grant from FRM. CHo received a CIFRE (Conventions Industrielles de Formation par la Recherche) scholarship in collaboration with the IRIS. HS received funding from the European Research Council (ERC) under the European Union's Horizon 2020 Research and Innovation Programme (ERC-2016-StG-71577).

Authors' Contributions: LW and DC: contributed equally to this work for the study concept and design, acquisition, analysis, and interpretation of data, and drafting of the manuscript. CHu, MSp, CHo, VLLM, VP, GF, NT and MSt: technical support. MD and FMN: histological analysis. SD and GK: fecal metabolite quantification. CSV: provided patients. HS: provided 
1 AhR KO mice. HS and PE: tryptophan metabolite analysis. GP: critical revision of the 2 manuscript, obtained funding, and provided patients. AMC: study concept, design, and 3 supervision, analysis and interpretation of the data, drafting of the manuscript, and funding 4 raising. 


\section{REFERENCES}

1 Collaborators GBDA. Alcohol use and burden for 195 countries and territories, 19902016: a systematic analysis for the Global Burden of Disease Study 2016. Lancet 2018;392:1015-35.

2 Seitz HK, Bataller R, Cortez-Pinto H, Gao B, Gual A, Lackner C, et al. Alcoholic liver disease. Nat Rev Dis Primers 2018;4:16.

3 Chen Y, Yang F, Lu H, Wang B, Lei D, Wang Y, et al. Characterization of fecal microbial communities in patients with liver cirrhosis. Hepatology 2011;54:562-72.

4 Ferrere G, Wrzosek L, Cailleux F, Turpin W, Puchois V, Spatz M, et al. Fecal microbiota manipulation prevents dysbiosis and alcohol-induced liver injury in mice. J Hepatol 2017;66:806-15.

5 Llopis M, Cassard AM, Wrzosek L, Boschat L, Bruneau A, Ferrere G, et al. Intestinal microbiota contributes to individual susceptibility to alcoholic liver disease. Gut 2016;65:8309.

6 Yan AW, Fouts DE, Brandl J, Starkel P, Torralba M, Schott E, et al. Enteric dysbiosis associated with a mouse model of alcoholic liver disease. Hepatology 2011;53:96-105.

7 Duan Y, Llorente C, Lang S, Brandl K, Chu H, Jiang L, et al. Bacteriophage targeting of gut bacterium attenuates alcoholic liver disease. Nature 2019;575:505-11.

8 Llorente C, Jepsen P, Inamine T, Wang L, Bluemel S, Wang HJ, et al. Gastric acid suppression promotes alcoholic liver disease by inducing overgrowth of intestinal Enterococcus. Nat Commun 2017;8:837.

9 Philips CA, Pande A, Shasthry SM, Jamwal KD, Khillan V, Chandel SS, et al. Healthy Donor Fecal Microbiota Transplantation in Steroid-Ineligible Severe Alcoholic Hepatitis: A Pilot Study. Clin Gastroenterol Hepatol 2017;15:600-2.

10 Grander C, Adolph TE, Wieser V, Lowe P, Wrzosek L, Gyongyosi B, et al. Recovery of ethanol-induced Akkermansia muciniphila depletion ameliorates alcoholic liver disease. Gut 2017.

11 Seo B, Jeon K, Moon S, Lee K, Kim WK, Jeong H, et al. Roseburia spp. Abundance Associates with Alcohol Consumption in Humans and Its Administration Ameliorates Alcoholic Fatty Liver in Mice. Cell Host Microbe 2020;27:25-40 e6.

12 Bajaj JS. Alcohol, liver disease and the gut microbiota. Nat Rev Gastroenterol Hepatol 2019;16:235-46.

13 Cassard AM, Ciocan D. Microbiota, a key player in alcoholic liver disease. Clin Mol Hepatol 2018;24:100-7.

14 Rao R. Endotoxemia and gut barrier dysfunction in alcoholic liver disease. Hepatology 2009;50:638-44.

15 Chen P, Starkel P, Turner JR, Ho SB, Schnabl B. Dysbiosis-induced intestinal inflammation activates TNFRI and mediates alcoholic liver disease in mice. Hepatology 2015;61:883-94.

16 Hartmann P, Chen P, Wang HJ, Wang L, McCole DF, Brandl K, et al. Deficiency of intestinal mucin-2 ameliorates experimental alcoholic liver disease in mice. Hepatology 2013;58:108-19.

17 Yoseph BP, Breed E, Overgaard CE, Ward CJ, Liang Z, Wagener ME, et al. Chronic alcohol ingestion increases mortality and organ injury in a murine model of septic peritonitis. PLoS One 2013;8: e62792.

18 Bajaj JS, Hylemon PB. Gut-liver axis alterations in alcoholic liver disease: Are bile acids the answer? Hepatology 2018;67:2074-5.

19 Hartmann P, Hochrath K, Horvath A, Chen P, Seebauer CT, Llorente C, et al. Modulation of the intestinal bile acid/farnesoid $\mathrm{X}$ receptor/fibroblast growth factor 15 axis improves alcoholic liver disease in mice. Hepatology 2018;67:2150-66. 
20 Lee JS, Cella M, McDonald KG, Garlanda C, Kennedy GD, Nukaya M, et al. AHR drives the development of gut ILC22 cells and postnatal lymphoid tissues via pathways dependent on and independent of Notch. Nat Immunol 2011;13:144-51.

21 Zelante T, Iannitti RG, Cunha C, De Luca A, Giovannini G, Pieraccini G, et al. Tryptophan catabolites from microbiota engage aryl hydrocarbon receptor and balance mucosal reactivity via interleukin-22. Immunity 2013;39:372-85.

22 Zheng Y, Valdez PA, Danilenko DM, Hu Y, Sa SM, Gong Q, et al. Interleukin-22 mediates early host defense against attaching and effacing bacterial pathogens. Nat Med 2008;14:282-9.

23 Badawy AA, Morgan CJ, Lovett JW, Bradley DM, Thomas R. Decrease in circulating tryptophan availability to the brain after acute ethanol consumption by normal volunteers: implications for alcohol-induced aggressive behaviour and depression. Pharmacopsychiatry 1995;28 Suppl 2:93-7.

24 Wrzosek L, Ciocan D, Borentain P, Spatz M, Puchois V, Hugot C, et al. Transplantation of human microbiota into conventional mice durably reshapes the gut microbiota. Sci Rep 2018;8:6854.

25 Williams GD, Proudfit AH, Quinn EA, Campbell KE. Variations in quantity-frequency measures of alcohol consumption from a general population survey. Addiction 1994;89:41320.

26 Alcoholic liver disease: morphological manifestations. Review by an international group. Lancet 1981;1:707-11.

27 Ciocan D, Voican CS, Wrzosek L, Hugot C, Rainteau D, Humbert L, et al. Bile acid homeostasis and intestinal dysbiosis in alcoholic hepatitis. Aliment Pharmacol Ther 2018.

28 Le Roy T, Debedat J, Marquet F, Da-Cunha C, Ichou F, Guerre-Millo M, et al. Comparative Evaluation of Microbiota Engraftment Following Fecal Microbiota Transfer in Mice Models: Age, Kinetic and Microbial Status Matter. Front Microbiol 2018;9:3289.

29 Dongowski G, Lorenz A, Proll J. The degree of methylation influences the degradation of pectin in the intestinal tract of rats and in vitro. The Journal of nutrition 2002;132:1935-44.

30 Cummings JH, Macfarlane GT, Englyst HN. Prebiotic digestion and fermentation. Am J Clin Nutr 2001;73:415S-20S.

31 Mathurin P, Deng QG, Keshavarzian A, Choudhary S, Holmes EW, Tsukamoto H. Exacerbation of alcoholic liver injury by enteral endotoxin in rats. Hepatology 2000;32:100817.

32 Wang L, Fouts DE, Starkel P, Hartmann P, Chen P, Llorente C, et al. Intestinal REG3 Lectins Protect against Alcoholic Steatohepatitis by Reducing Mucosa-Associated Microbiota and Preventing Bacterial Translocation. Cell Host Microbe 2016;19:227-39.

33 Langille MG, Zaneveld J, Caporaso JG, McDonald D, Knights D, Reyes JA, et al. Predictive functional profiling of microbial communities using 16S rRNA marker gene sequences. Nat Biotechnol 2013;31:814-21.

34 Hayes MD, Ovcinnikovs V, Smith AG, Kimber I, Dearman RJ. The aryl hydrocarbon receptor: differential contribution to $\mathrm{T}$ helper 17 and $\mathrm{T}$ cytotoxic 17 cell development. PLoS One 2014;9:e106955.

35 Postler TS, Ghosh S. Understanding the Holobiont: How Microbial Metabolites Affect Human Health and Shape the Immune System. Cell Metab 2017;26:110-30.

36 Chen P, Torralba M, Tan J, Embree M, Zengler K, Starkel P, et al. Supplementation of Saturated Long-Chain Fatty Acids Maintains Intestinal Eubiosis and Reduces Ethanol-induced Liver Injury in Mice. Gastroenterology 2015;148:203-14 e16.

37 Hendrikx T, Duan Y, Wang Y, Oh JH, Alexander LM, Huang W, et al. Bacteria engineered to produce IL-22 in intestine induce expression of REG3G to reduce ethanolinduced liver disease in mice. Gut 2018. 
38 Bull-Otterson L, Feng W, Kirpich I, Wang Y, Qin X, Liu Y, et al. Metagenomic analyses of alcohol induced pathogenic alterations in the intestinal microbiome and the effect of Lactobacillus rhamnosus GG treatment. PLoS One 2013;8:e53028.

39 Chen RC, Xu LM, Du SJ, Huang SS, Wu H, Dong JJ, et al. Lactobacillus rhamnosus GG supernatant promotes intestinal barrier function, balances Treg and TH17 cells and ameliorates hepatic injury in a mouse model of chronic-binge alcohol feeding. Toxicol Lett 2016;241:103-10.

40 Wang Y, Liu Y, Kirpich I, Ma Z, Wang C, Zhang M, et al. Lactobacillus rhamnosus GG reduces hepatic TNFalpha production and inflammation in chronic alcohol-induced liver injury. J Nutr Biochem 2013;24:1609-15.

41 Ciocan D, Rebours V, Voican CS, Wrzosek L, Puchois V, Cassard AM, et al. Characterization of intestinal microbiota in alcoholic patients with and without alcoholic hepatitis or chronic alcoholic pancreatitis. Sci Rep 2018;8:4822.

42 Shin NR, Whon TW, Bae JW. Proteobacteria: microbial signature of dysbiosis in gut microbiota. Trends Biotechnol 2015;33:496-503.

43 Singh V, Yeoh BS, Chassaing B, Xiao X, Saha P, Aguilera Olvera R, et al. Dysregulated Microbial Fermentation of Soluble Fiber Induces Cholestatic Liver Cancer. Cell 2018;175:679$94 \mathrm{e} 22$.

44 Medicine Institute. Dietary Reference Intakes for Energy, Carbohydrate, Fiber, Fat, Fatty Acids, Cholesterol, Protein, and Amino Acids. 2005.

45 Davies GJ, Crowder M, Dickerson JW. Dietary fibre intakes of individuals with different eating patterns. Hum Nutr Appl Nutr 1985;39:139-48.

46 Buscail C, Bourcier V, Fezeu LK, Roulot D, Brule S, Ben-Abdesselam Z, et al. Eating Patterns in Patients with Compensated Cirrhosis: A Case-Control Study. Nutrients 2018;10.

47 Alferink LJM, Erler NS, de Knegt RJ, Janssen HLA, Metselaar HJ, Darwish Murad S, et al. Adherence to a plant-based, high-fibre dietary pattern is related to regression of nonalcoholic fatty liver disease in an elderly population. Eur J Epidemiol 2020.

48 Partula V, Deschasaux M, Druesne-Pecollo N, Latino-Martel P, Desmetz E, Chazelas $\mathrm{E}$, et al. Associations between consumption of dietary fibers and the risk of cardiovascular diseases, cancers, type 2 diabetes, and mortality in the prospective NutriNet-Sante cohort. Am J Clin Nutr 2020;112:195-207.

49 Seyrig JA, Naveau S, Gonzales R, Petit R. Pectines. Gastroenterol Clin Biol 1983;7:1031-37.

50 Ralphs DNL, Lawaetz NJG. Effect of dietary fibre on gastric emptying in dumpers. Gut 1978;19:986-87.

51 Ki SH, Park O, Zheng M, Morales-Ibanez O, Kolls JK, Bataller R, et al. Interleukin-22 treatment ameliorates alcoholic liver injury in a murine model of chronic-binge ethanol feeding: role of signal transducer and activator of transcription 3. Hepatology 2010;52:1291-300.

52 Lamas B, Richard ML, Leducq V, Pham HP, Michel ML, Da Costa G, et al. CARD9 impacts colitis by altering gut microbiota metabolism of tryptophan into aryl hydrocarbon receptor ligands. Nat Med 2016;22:598-605.

53 Natividad JM, Agus A, Planchais J, Lamas B, Jarry AC, Martin R, et al. Impaired Aryl Hydrocarbon Receptor Ligand Production by the Gut Microbiota Is a Key Factor in Metabolic Syndrome. Cell Metab 2018.

54 Wang X, Ota N, Manzanillo P, Kates L, Zavala-Solorio J, Eidenschenk C, et al. Interleukin-22 alleviates metabolic disorders and restores mucosal immunity in diabetes. Nature 2014;514:237-41.

55 Yan J, Tung HC, Li S, Niu Y, Garbacz WG, Lu P, et al. Aryl Hydrocarbon Receptor Signaling Prevents Activation of Hepatic Stellate Cells and Liver Fibrogenesis in Mice. Gastroenterology 2019. 
156 Mackos AR, Galley JD, Eubank TD, Easterling RS, Parry NM, Fox JG, et al. Social 2 stress-enhanced severity of Citrobacter rodentium-induced colitis is CCL2-dependent and 3 attenuated by probiotic Lactobacillus reuteri. Mucosal Immunol 2016;9:515-26.

457 Ahl D, Liu H, Schreiber O, Roos S, Phillipson M, Holm L. Lactobacillus reuteri 5 increases mucus thickness and ameliorates dextran sulphate sodium-induced colitis in mice. 6 Acta Physiol (Oxf) 2016;217:300-10.

758 Aoki R, Aoki-Yoshida A, Suzuki C, Takayama Y. Indole-3-Pyruvic Acid, an Aryl 8 Hydrocarbon Receptor Activator, Suppresses Experimental Colitis in Mice. J Immunol 9 2018;201:3683-93.

1059 Naganuma M. Treatment with indigo naturalis for inflammatory bowel disease and other 11 immune diseases. Immunol Med 2019:1-6. 


\section{FIGURE LEGENDS}

Figure 1. Altering the intestinal microbiota using pectin reverses alcohol induced liver lesions. Alc, alcohol-fed mice; Ctrl, control-fed mice; Alc $\mathrm{F}_{1}$, alcohol-fed mice humanized with the microbiota from a patient with severe alcoholic hepatitis $\left(\mathrm{sAH}\right.$, patient $\left.\mathrm{F}_{1}\right)$; Alc $\mathrm{F}_{1} \mathrm{P}$, alcohol-fed mice humanized with microbiota from a patient with $\mathrm{sAH}$ (patient $\mathrm{F}_{1}$ ) and treated with $6.5 \%$ pectin. (a) Experimental design: mice were progressively adapted to a semi-liquid, Lieber DeCarli (LDC) diet, then an ethanol diet (1-3\%), and finally fed a 5\% ethanol diet for one week. Pectin was introduced in the diet at the same time as the 5\% ethanol. Microbiota analysis: (b) PCoA plot, showing the unweighted UniFrac distance $(\mathrm{p}<0.001, \mathrm{R}=0.58$, ANOSIM test, 10,000 permutations, using the first 5 PC); (c) LDA effect size (LEfSe) cladograms showing the taxa most differentially associated with Alc $\mathrm{F}_{1}$ (red) or Alc $\mathrm{F}_{1} \mathrm{P}$ mice (yellow) (Wilcoxon rank-sum test). Circle sizes in the cladogram plot are proportional to bacterial abundance. The circles represent, going from the inner to outer circle: phyla, genus, class, order, and family. (d) ALT level in Ctrl $(\mathrm{n}=8)$, Alc $(\mathrm{n}=8)$, Alc $\mathrm{F}_{1}(\mathrm{n}=12)$, and Alc $\mathrm{F}_{1} \mathrm{P}$ $(\mathrm{n}=10)$ mice. (e) Liver triglyceride quantification in Ctrl $(\mathrm{n}=8)$, Alc $(\mathrm{n}=8), \operatorname{Alc} \mathrm{F}_{1}(\mathrm{n}=12)$, and Alc $F_{1} P(n=10)$ mice. (f) Representative images of liver sections stained with hematoxylineosin, scale bar $100 \mu \mathrm{m}$. (g) Liver mRNA levels determined by qPCR: $c c l 2, \operatorname{tnf} \alpha$, ill $\beta$, and $c c l 3$ normalized to that of the gapdh gene in $\operatorname{Ctrl}(\mathrm{n}=4)$, Alc $(\mathrm{n}=5), \mathrm{Alc}_{1}(\mathrm{n}=12)$, and Alc $\mathrm{F}_{1} \mathrm{P}$ $(n=10)$ mice. Results $(d-g)$ are shown as the mean \pm SEM. Significant results for $* p<0.05$, $* * \mathrm{p}<0.01$, and $* * * \mathrm{p}<0.001$ were determined by Mann-Whitney tests unless stated otherwise.

Figure 2. Functions of the intestinal microbiota and fecal metabolome are modified by pectin treatment. Ctrl, control-fed mice; Alc, alcohol-fed mice; Alc $\mathrm{F}_{1}$ and $\mathrm{Alc} \mathrm{F}_{3}$, alcohol-fed mice humanized with microbiota from a patient with sAH (patients $\mathrm{F}_{1}$ or $\mathrm{F}_{3}$ ); Alc $\mathrm{F}_{1} \mathrm{P}$ and Alc $\mathrm{F}_{3} \mathrm{P}$, alcohol-fed mice humanized with microbiota from a patient with sAH (patients $\mathrm{F}_{1}$ or $\mathrm{F}_{3}$ ) and treated with $6.5 \%$ pectin $\left(\mathrm{F}_{1}\right)$ or $2 \%$ pectin $\left(\mathrm{F}_{3}\right)$. (a) LEfSe cladograms of KEGG pathway contributions of predicted metagenomic data in Alc $\mathrm{F}_{1}$ and $\mathrm{Alc} \mathrm{F}_{1} \mathrm{P}$ mice (Wilcoxon rank-sum test). In a: $\operatorname{Ctrl} \mathrm{n}=8$, Alc $\mathrm{n}=10$, Alc $\mathrm{F}_{1} \mathrm{n}=14$, and Alc $\mathrm{F}_{1} \mathrm{P} \mathrm{n}=10$ mice per experiment. (b) PCA ordination plot on all fecal metabolomic data (147 metabolites). (c) Heatmap showing the first 60 metabolites ranked by t-tests between Alc $F_{1}$ (red) and Alc $F_{1} P$ (green). (d) Metabolic enrichment analysis showing the most altered pathways in Alc $\mathrm{F}_{1} \mathrm{P}$ mice relative to Alc $\mathrm{F}_{1}$ mice. All matched pathways are displayed as circles. The color and size of each circle are based on the $\mathrm{p}$ value and pathway impact value, respectively. The graph was obtained by plotting the 
$-\log$ of $p$ values from the pathway enrichment analysis on the $y$ axis and the pathway impact values, derived from the pathway topology analysis, on the $\mathrm{x}$ axis. (e) Relative fecal levels of tryptophan, kynurenine, and indole. In b-e: $\operatorname{Ctrl} n=8$, Alc $n=10$, Alc $F_{1} n=11$, and Alc $F_{1} P n$ $=7$ mice per group. (f) Tryptophan metabolites quantification in faeces. Aryl hydrocarbon receptor (AhR) ligands (methyl-indole 3-acetic acid, indole 3-propionic acid, indole 3aldehyde, indole 3-acrylic acid, and 3-indoxyl sulfuric acid). In f: $\operatorname{Ctrl} n=8$, Alc n $=9$, Alc F 3 $\mathrm{n}=6$, and $\mathrm{Alc}_{3} \mathrm{P} \mathrm{n}=8$ mice per group. ${ }^{*} \mathrm{p}<0.05$, ${ }^{*} \mathrm{p}<0.01, * * * \mathrm{p}<0.001$ by the ANOVA or Kruskal-Wallis test with Tukey or Dunn correction for multiple comparisons, as appropriated.

Figure 3. AhR activation reverses alcohol-induced liver lesions. Ctrl, control-fed mice; Alc, alcohol-fed mice. (a,b) Alc $\mathrm{F}_{3}$, alcohol-fed mice humanized with the microbiota from a patient with sAH (patient $\mathrm{F}_{3}$ ); Alc $\mathrm{F}_{3} \mathrm{P} 2$, Alc $\mathrm{F}_{3}$ P6.5, alcohol-fed mice humanized with the microbiota from a patient with sAH (patient $\mathrm{F}_{3}$ ) and treated with 2 or $6.5 \%$ pectin. Colon mRNA levels were determined by qPCR: (a) cyplal and ahrr, (b) ill 7 and il22, normalized to that of the $18 \mathrm{~s}$

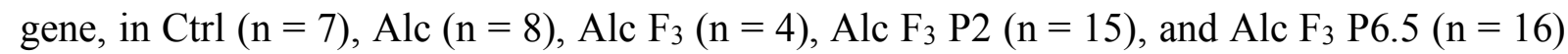
mice. (c-g) DMSO or Ficz, mice treated with DMSO or Ficz. (c) Liver cyplal and scdl, normalized to that of the gapdh gene. (d) ALT level and liver triglyceride quantification in Ctrl DMSO $(\mathrm{n}=7)$, Alc DMSO $(\mathrm{n}=12)$, Ctrl Ficz $(\mathrm{n}=8)$, and Alc Ficz $(\mathrm{n}=8)$ mice. (e) Liver mRNA levels determined by qPCR: $\operatorname{ccl} 2$ and $\operatorname{tnf} \alpha$, normalized to that of the gapdh gene, in Ctrl DMSO $(\mathrm{n}=6)$, Alc DMSO $(\mathrm{n}=11)$, Ctrl Ficz $(\mathrm{n}=8)$, and Alc Ficz $(\mathrm{n}=7)$ mice. $(\mathrm{f})$ Colon mRNA levels determined by qPCR: reg3 $\beta$ and $\operatorname{reg} 3 \gamma$, normalized to that of the gapdh gene. (g) Ileum mRNA levels determined by qPCR: $\operatorname{reg} 3 \beta$ and $\operatorname{reg} 3 \gamma$, normalized to that of the gapdh gene, in Ctrl DMSO ( $\mathrm{n}=8)$, Alc DMSO $(\mathrm{n}=12)$, Ctrl Ficz $(\mathrm{n}=8)$, and Alc Ficz $(\mathrm{n}=10)$ mice.

\section{Figure 4. KO of AhR partly blocks the liver protective effects of pectin.}

(a-d) WT, wild type mice; AhR KO, AhR deficient mice; Alc P2, alcohol-fed mice treated with $2 \%$ pectin. (a) ALT levels and liver triglyceride quantification in WT Ctrl $(\mathrm{n}=8)$, WT Alc (n $=5)$, WT Alc P $(\mathrm{n}=8)$, AhR KO Ctrl $(\mathrm{n}=4)$, AhR KO Alc $(\mathrm{n}=4)$, and AhR KO Alc P $(\mathrm{n}=5)$ mice. (b) Representative images of liver sections stained with hematoxylin-eosin, scale bar 100 $\mu \mathrm{m}$. (c,d) Colon mRNA levels determined by qPCR: cyplal, ahr, il22, reg3 $\beta$ and reg3 $\gamma$, normalized to that of the $18 s$ gene, in WT Ctrl $(\mathrm{n}=8)$, WT Alc $(\mathrm{n}=5)$, WT Alc P $(\mathrm{n}=8)$, AhR KO Ctrl $(n=4)$, AhR KO Alc $(n=4)$, and AhR KO Alc P $(n=5)$ mice. Results are shown as the mean \pm SEM. Significant results for $* p<0.05$, $* * p<0.01$, and $* * * p<0.001$ were determined by Mann-Whitney tests unless stated otherwise. 
2 Figure 5. Tryptophan metabolism is reduced in patients with severe alcoholic hepatitis

3 (sAH) and correlates with disease severity. (a) Serum concentrations of tryptophan, 4 kynurenine, and AHR agonists (tryptamine, indole, indole 3-acetic acid, indole 3-acetaldehyde, 5 and indoxyl sulfate) in sAH patients $(\mathrm{n}=14)$ and patients without severe alcoholic hepatitis 6 (noAH, $\mathrm{n}=15)$. (b) Spearman correlation of the serum tryptophan and AST, bilirubin, and 7 prothrombin time levels and MELD score.

8 
Figure 1

a

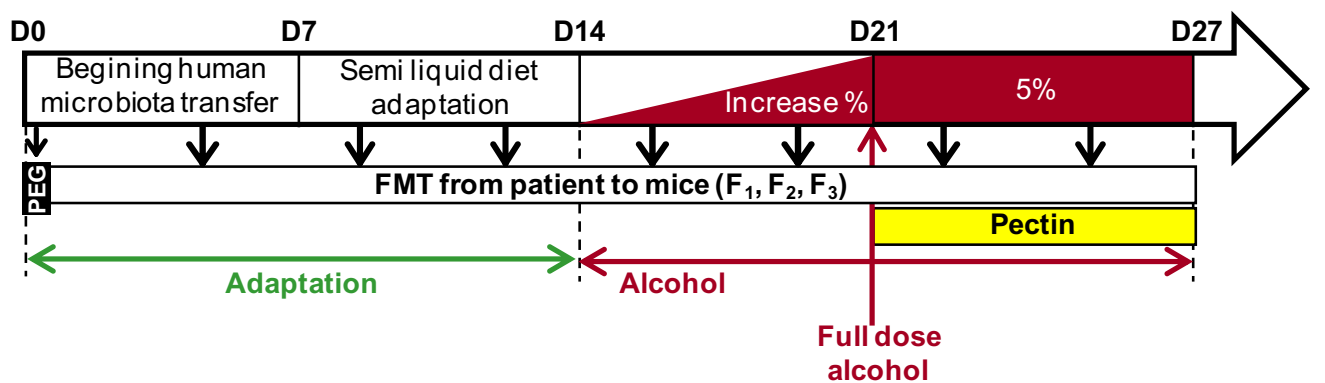

b

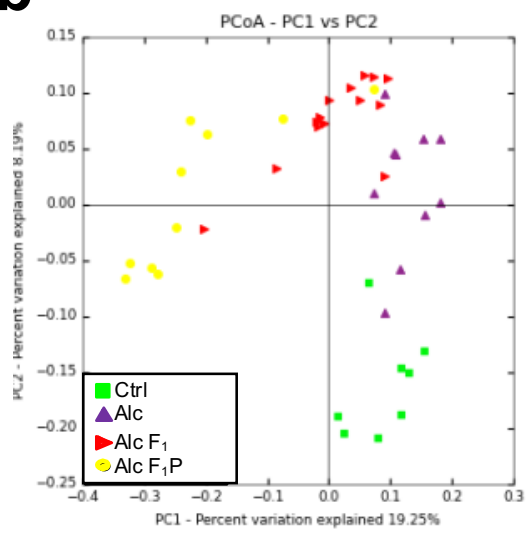

d

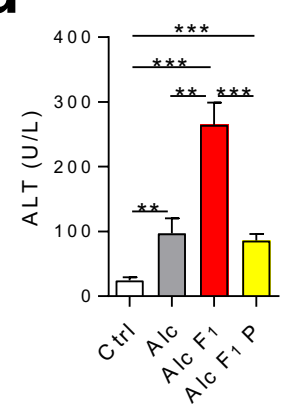

e

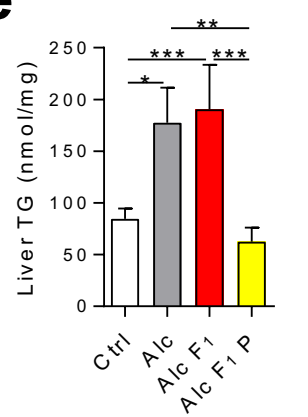

f

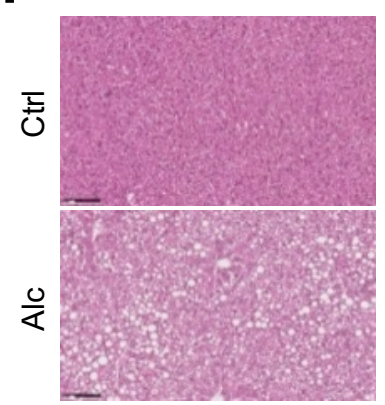

$\frac{0}{\frac{0}{4}}$

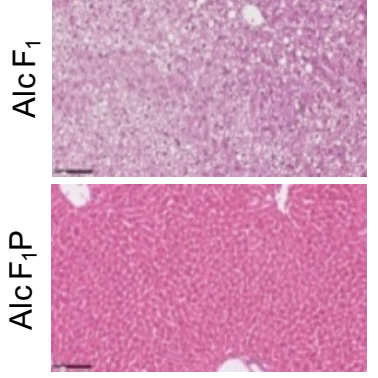

C
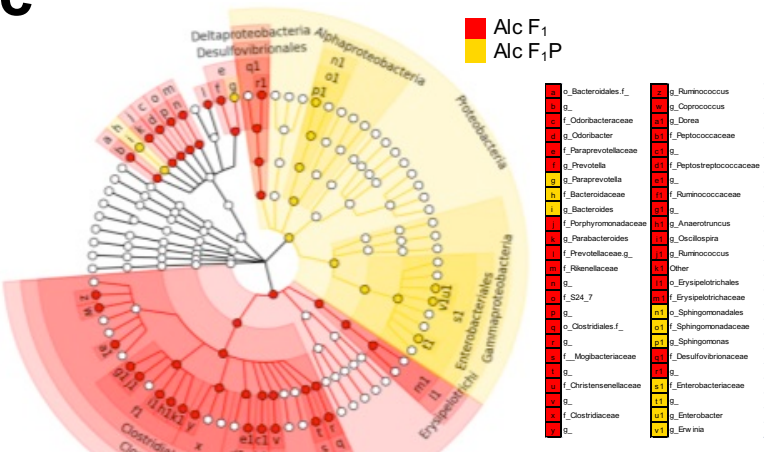

g
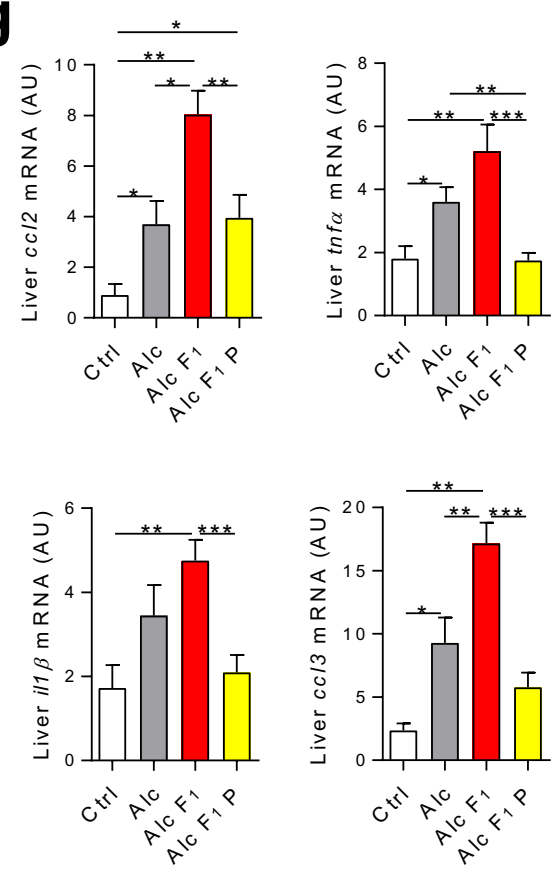


\section{Figure 2}

a
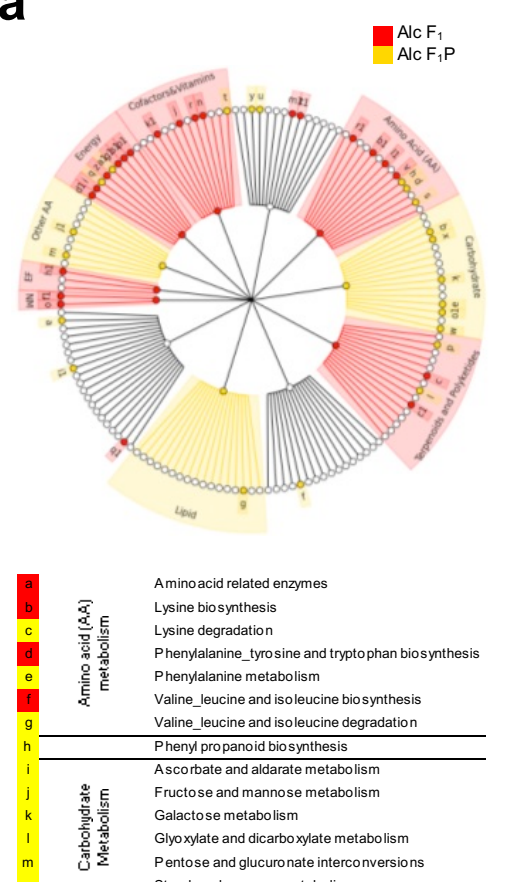

$\begin{array}{ccl}m & \mathcal{O}^{\mathrm{m}} & \begin{array}{l}\text { Pentose and glucuronate interconversions } \\ \text { Starch and sucrose metabolism }\end{array} \\ \mathrm{n} & & \begin{array}{l}\text { Carbon fixation in photosynthetic organisms } \\ \text { Carbon fixation pathways in prokaryotes }\end{array} \\ \mathrm{p} & \mathrm{E} & \end{array}$

Methane metabolism

Nitrogenmetabolism

Oxidative phosphorylation

Photosynthesis

Photosynthesis protein

Sulfur metabolism

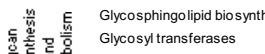

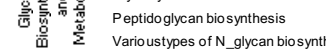

\begin{tabular}{|c|c|}
\hline \multicolumn{2}{|c|}{ b1 Lipid Metabolism Biosynth unsatFA } \\
\hline & \\
\hline & Onecarbonpoolbyfolate \\
\hline 要 & PantothenateandCoAbiosynthesis \\
\hline & Thiamine metabolism \\
\hline & Ubiquinoneandotherterpenoid \\
\hline h1 Metabolism of & Cyanoaminoacid \\
\hline Other AA & Glutathione \\
\hline & Biosynth nonribosomal peptides \\
\hline 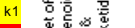 & Geraniol degradation \\
\hline & Prenyttransferases \\
\hline & Terpenoidbackbone biosynt \\
\hline Nucleotide Met & Purine metabolism \\
\hline (NM) & Pyrimidine metabolism \\
\hline 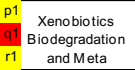 & $\begin{array}{l}\text { Drug met cyp } 450 \\
\text { Drug met } \\
\text { Met xenobiotics cyp } 450\end{array}$ \\
\hline
\end{tabular}

b

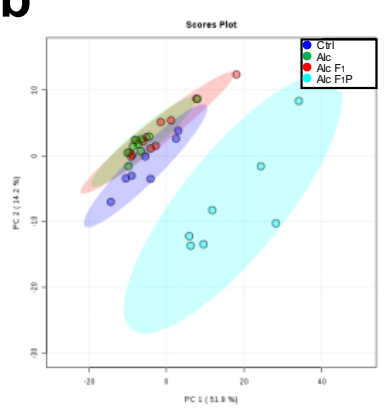

d

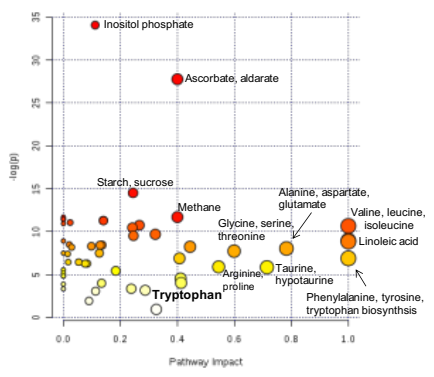

e

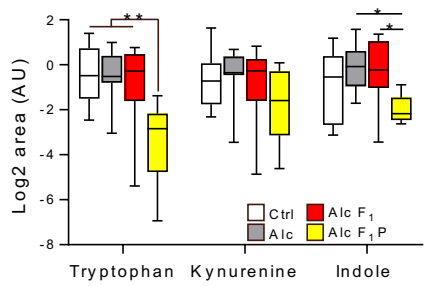

f

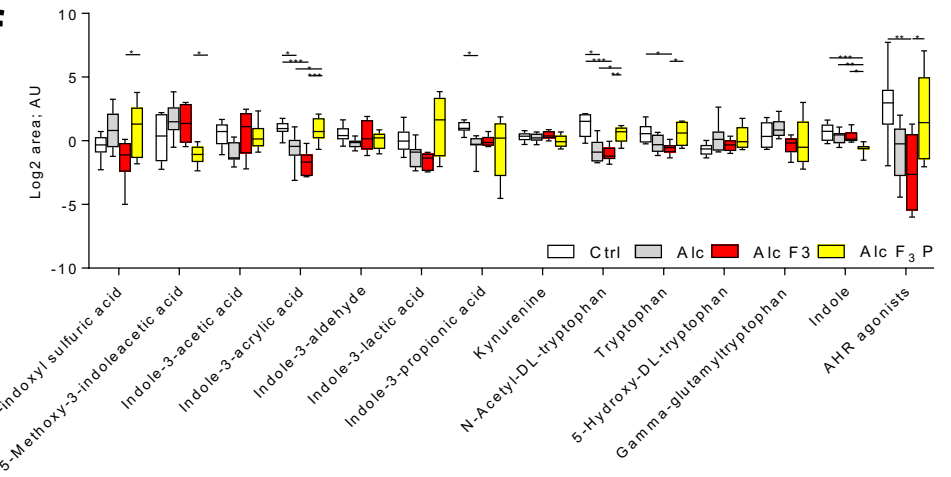

C

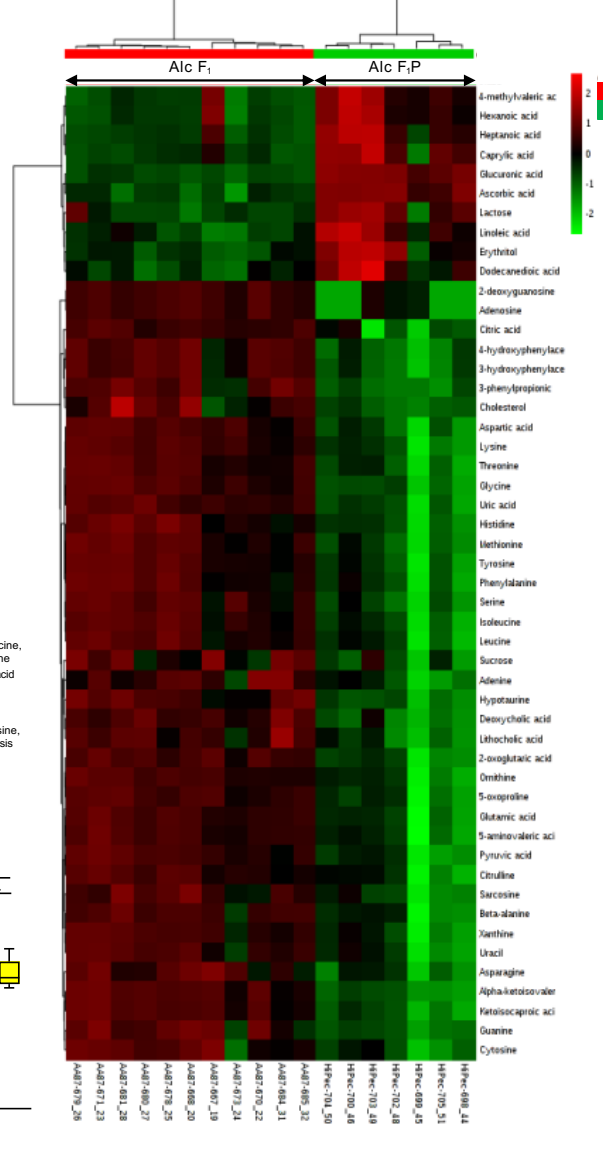


Figure 3
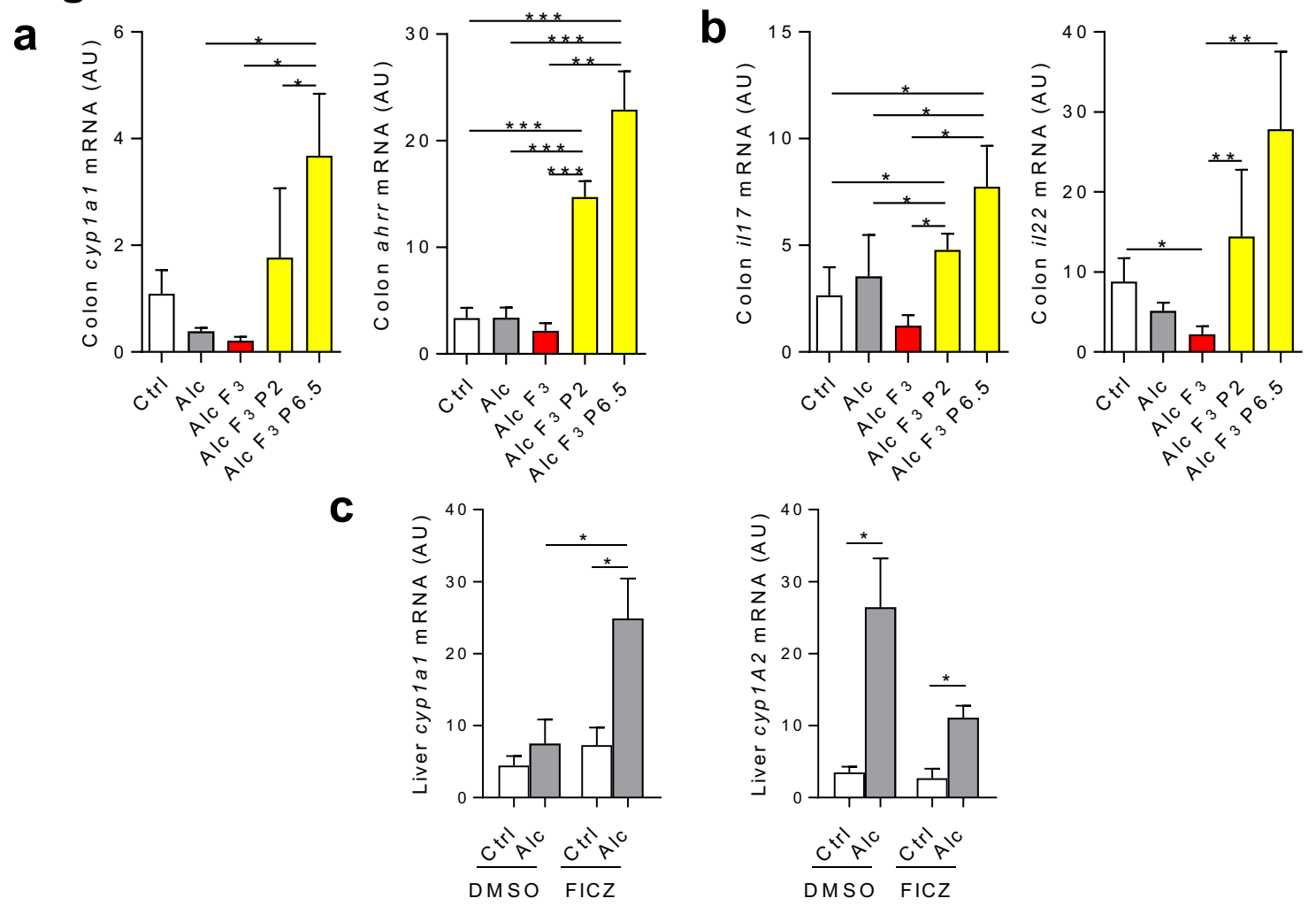

d

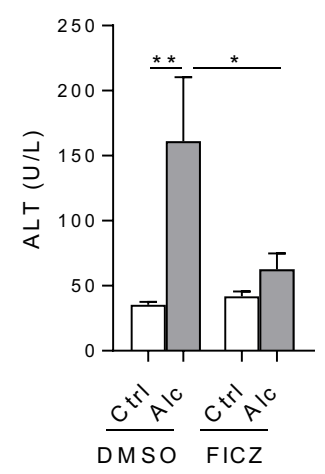

f

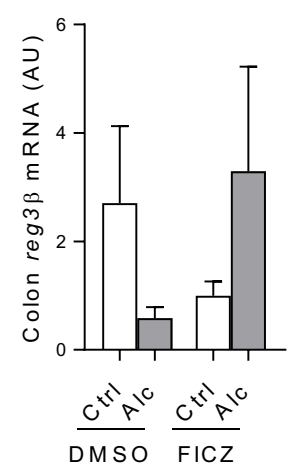

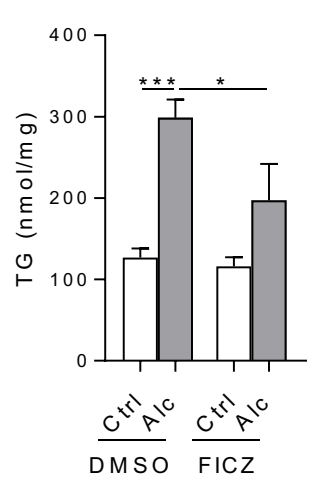

e

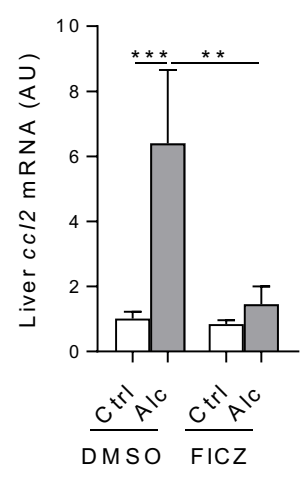

g
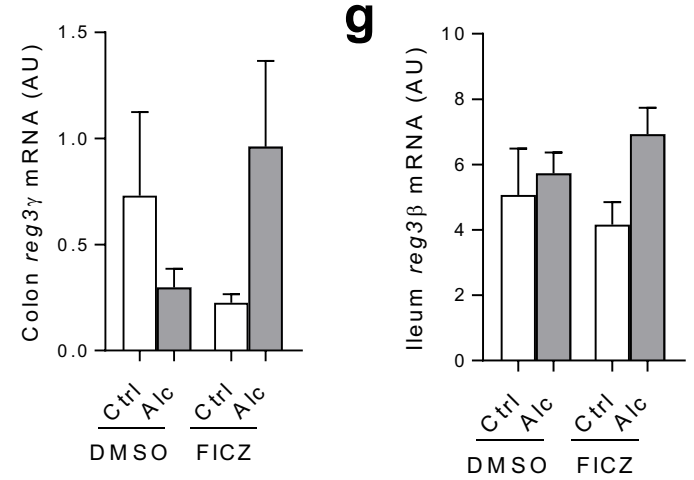

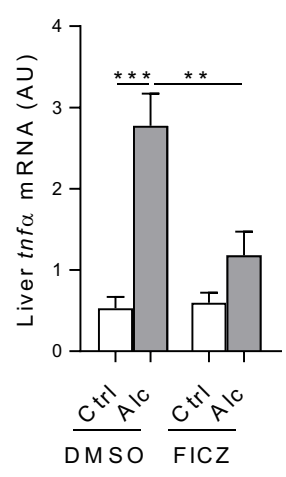




\section{Figure 4}

a

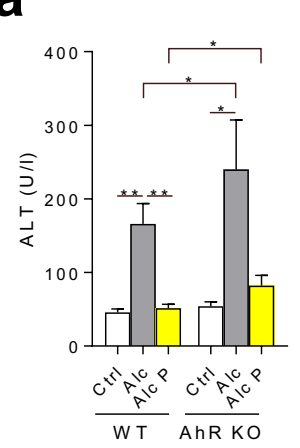

C

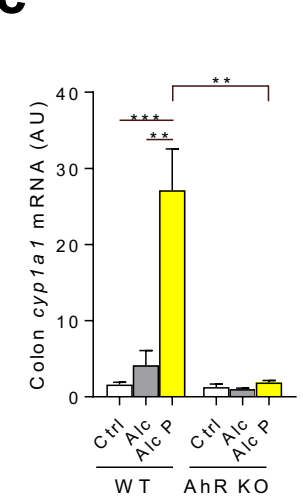

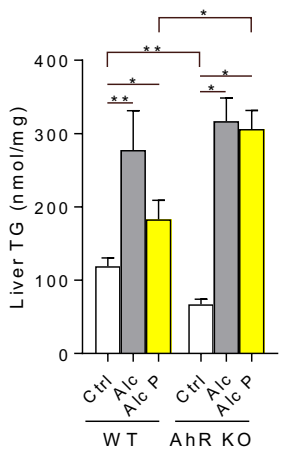

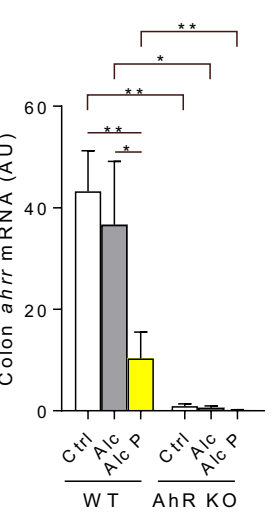

b

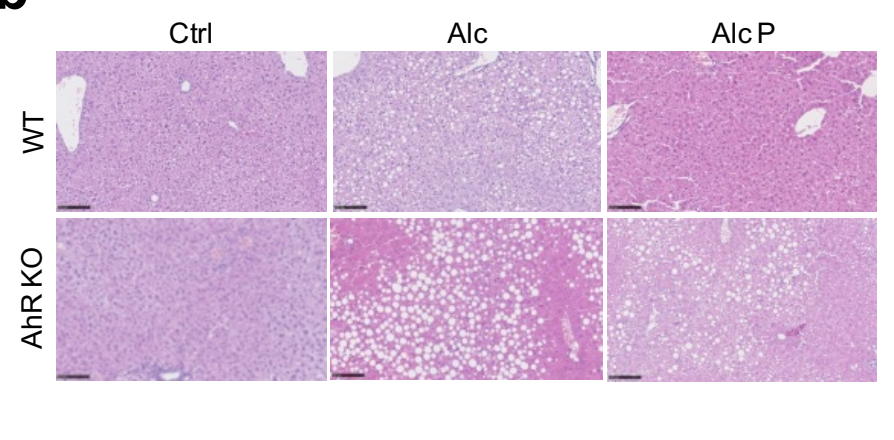

d
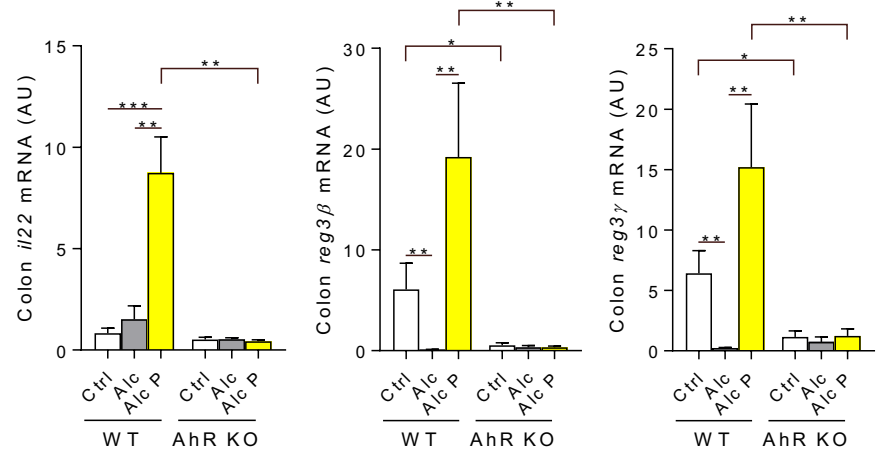

1

2 


\section{Figure 5}

a
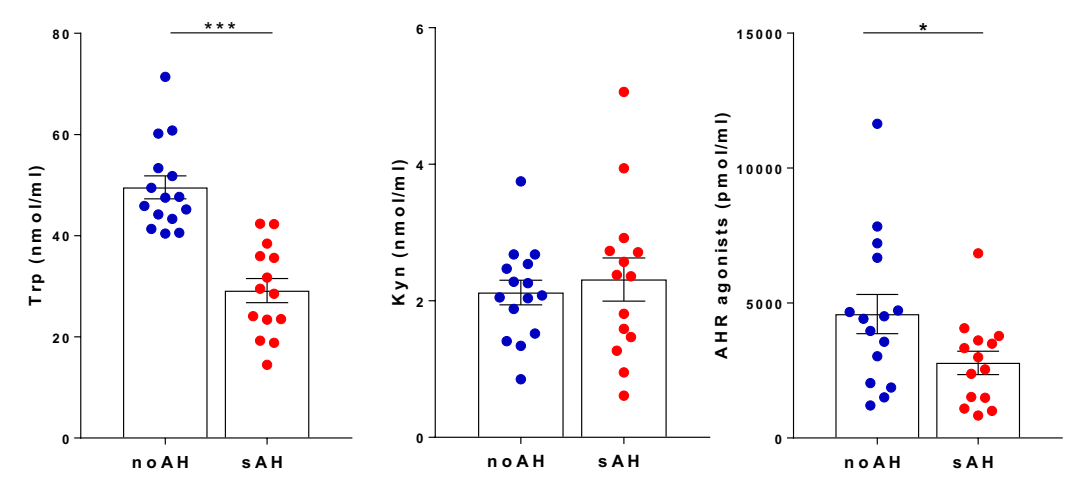

b
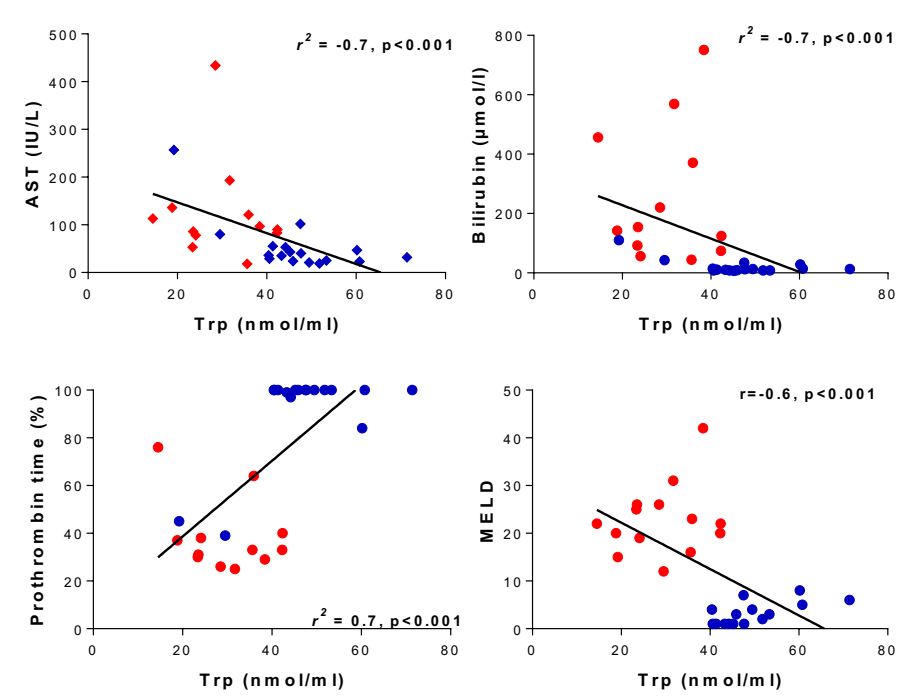

1 
1

2

3

4

5

6

7

8

9

10

11

12

13

14

15

16

17

18

\section{SUPPLEMENTARY DATA}

Material and methods

4

Supplementary Figure 1

Supplementary Figure 2 11

Supplementary Figure 3 12

Supplementary Figure 4

Supplementary Figure 5

Supplementary Figure 6

Supplementary Figure 7 16

Supplementary Figure 8 17

Supplementary Table $1 \quad 18$

Supplementary Table 2 19

Supplementary Table 3

Supplementary Table $4 \quad 24$

Supplementary Table 5 


\section{MATERIAL AND METHODS}

Chronic exposure to alcohol. Eight-week old mice were fed a liquid diet adapted from Lieber DeCarli for 21 days, as previously described based on the NIAAA model ${ }^{3}$ but without a binge administration of alcohol at the end. Briefly, the ethanol diet was obtained by adding absolute ethanol to a solution of Lieber DeCarli powder (Ssniff, Spezialdiäten GmbH, Soest, Germany) in filtered water. After a 7-day period of adaptation to the animal facility and a 7-day period adaptation to the semi-liquid diet, mice were given increasing amounts of ethanol for 7 days ( $1 \%$ increase every two days). The final concentration of ethanol in this liquid diet was $5 \%$ ( $\mathrm{vol} / \mathrm{vol})$, such that ethanol accounted for $28 \%$ of the total caloric intake. The control diet was obtained by replacing the ethanol with an isocaloric amount of maltodextrin (Maldex 170, Safe, France). Alcohol-fed groups were allowed free access to the 5\% (vol/vol) ethanol diet for 7 days. Control mice were fed the isocaloric control diet throughout the entire feeding period. During the Lieber DeCarli diet, animals did not have access to drinking water. Diet consumptions were recorded and were similar between the groups (data not shown).

Tissues and samples. Mice were anesthetized and blood samples collected in EDTA coated tubes. Liver and distal colon were excised: one piece was fixed in buffered formaldehyde and another piece was snap-frozen in liquid nitrogen for TG and RNA extractions. All samples were stored at $-80^{\circ} \mathrm{C}$ until use. Fecal samples were collected from mice immediately before euthanasia.

Measurement of bacterial translocation in the liver. Liver were collected in sterile conditions and disrupted in $2 \mathrm{ml}$ of PBS 1X. $500 \mu \mathrm{l}$ of lysate were put on PolyVitex chocolate agar (Biomérieux, Capronne, France), spread with balk and place in incubator in anaerobic conditions at $37^{\circ} \mathrm{C}$ during 48 to 72 hours and colony-forming unit were counted.

Measurement of liver triglycerides and blood samples. Liver triglycerides were extracted using a triglyceride quantification kit following the manufacturer's indications (Abcam, Cambridge, UK). Quantification was performed by using a Berthold Technologies colorimetric microplate reader (Mithras LB 940), and the level of liver TG was expressed in nmol per milligram of liver. Plasma alanine aminotransferase (ALT), aspartate transaminase (AST), triglycerides, highdensity lipoprotein (HDL) and cholesterol levels were assed using a spectrophotometric method 
1 (Olympus, AU400). Alcohol was measured in the plasma by using the colorimetric assay kit 2 (Biovision).

3

Liver and gut histology. Liver and gut (colon) were fixed overnight in 4\% paraformaldehyde and embedded in paraffin. Paraffin sections ( $4 \mu \mathrm{m}$ thick) were stained with hematoxylin and eosin (H\&E). Colon samples were also stained with Alcian Blue.

Immunofluorescence. Specimens were embedded in paraffin and cut in $3-\mu \mathrm{m}$ section. Staining with antibodies purchased from abcam against ZO-1 (ab96587) and occludine (ab216327) was done, followed by staining with a fluorochrome-coupled secondary antibody goat anti-rabbit Alexa FluorTM Plus 594 (Invitrogen, Thermo Fisher Scientific). Nuclei were stained with Hoechst (Life Technologies, Thermo Fisher Scientific). Slides were scanned by the digital slide scanner NanoZoomer 2.0-RS (Hamamatsu, France) allowing an overall view of the samples. Images were digitally captured from the scanned slides using the NDP.view2 software (Hamamatsu, France).

RNA extraction and quantification. Mice livers were disrupted in Qiazol solution. Total RNA was extracted using a Qiagen RNeasy Lipid tissue minikit (Courtaboeuf, France). Total gut RNA was extracted using a Qiagen RNeasy Plus Mini Kit (Courtaboeuf, France), after being disrupted with an MP Biomedicals FastPrep. The RNA integrity number (RIN) was determined using an Agilent Bioanalyzer 2100 system with the RNA 6000 Nano Labchip kit. Samples with a RIN of less than 8 were eliminated. For cDNA synthesis, $1 \mu \mathrm{g}$ of each total RNA sample was reverse transcribed. A $12 \mu \mathrm{lmix}$ containing $1 \mu \mathrm{g}$ of RNA, random hexamers (Roche Diagnostics, Meylan, France), and 10 mM dNTP Mix (Invitrogen, Carslbad, CA) was prepared for each sample. Mixtures were heated at $65^{\circ} \mathrm{C}$ for $5 \mathrm{~min}$, cooled on ice, and then an $8 \mu 1$ reaction mix containing $1 \mu \mathrm{l} \mathrm{M-MuLv} \mathrm{RT} \mathrm{(Invitrogen),} 4 \mu 1$ xx Buffer (Invitrogen), $2 \mu 10.1 \mathrm{M}$ dithiothreitol (Invitrogen), and $1 \mu 1$ Protector RNase Inhibitor (40 U/ $\mu 1$; Invitrogen) was added. The reaction conditions were $10 \mathrm{~min}$ at $25^{\circ} \mathrm{C}, 50 \mathrm{~min}$ at $50^{\circ} \mathrm{C}, 15 \mathrm{~min}$ at $70^{\circ} \mathrm{C}$.

Gene expression analysis by quantitative qPCR. Real-time qPCR was performed in a Light Cycler 480 (Roche Diagnostics) using the LC FastStart DNA Master SYBR Green I kit (Roche Diagnostics). Amplification was initiated with an enzyme activation step at $95^{\circ} \mathrm{C}$ for $10 \mathrm{~min}$, followed by 40 cycles consisting of a $20 \mathrm{~s}$ denaturation step at $95^{\circ} \mathrm{C}$, a $15 \mathrm{~s}$ annealing step at the temperature appropriate for each primer, and a $45 \mathrm{~s}$ elongation step at $72^{\circ} \mathrm{C}$. We amplified 
1 the cDNAs for $18 s$, gapdh, thf $\alpha, \operatorname{tg} f \beta, i l 1 \beta, c c l 2, c c l 3$, reg3b, reg3g, il22, ill 7 , ahrr and cyplal.

2 Primer sequences are listed in Supplemental Table 5. Data were analyzed using Light Cycler 480 Software (Roche Diagnostics). Relative gene expression was normalized to the $18 \mathrm{~s}$ or gapdh reference gene.

Analysis of the intestinal microbiota by $16 S \mathrm{RNA}$ sequencing. The composition of the microbiota was analyzed using Illumina MiSeq technology targeting the 16S ribosomal DNA V3-V4 region in paired-end modus ( 2 x 300 base pair) (GenoToul, Toulouse). Bacterial DNA was obtained by homogenizing stools in a Guanidinium thiocyanate containing lysis buffer using a Fast Prep homogenizer. High quality bacterial DNA was extracted by successive steps of purification and precipitation using "Laboratory-made" buffers ${ }^{4}$. PCR were performed to prepare amplicons using V3-V4 oligonucleotides (PCR1F_460: 5' CTTTCCCTACACGACGCTCTTCCGATCTACGGRAGGCAGCAG 3', PCR1R_460: 5' GGAGTTCAGACGTGTGCTCTTCCGATCTTACCAGGGTATCTAATCCT 3’). Amplicon quality was verified by gel electrophoresis and they were sent to the GenoToul plateform for sequencing. The resulting paired reads were assembled using PANDAseq v 2.7 to generate an amplicon size of 450 base pairs ${ }^{5}$. Reads were demultiplexed and processed using the quantitative insights into microbial ecology (QIIME v1.9.0) pipeline and the default parameters of QIIME ${ }^{6}$. Chimeric sequences were identified de novo, reference based, and then removed using usearch61 ${ }^{7}$. The non-chimeric sequences were then clustered into operational taxonomic units (OTUs) at $97.0 \%$ sequence similarity using a closed reference-based picking approach with UCLUST software against the Greengenes database 13_8 of bacterial 16S rDNA sequences ${ }^{8}$. The mean number of quality-controlled reads was $25034 \pm 6875$ (mean $\pm \mathrm{SD}$ ) per sample. After rarefaction at 7,000 reads per sample, bacterial alpha diversity was estimated using Shannon index. OTUs with a prevalence $<5 \%$ were removed from the analysis. Analyses using R software v2.14.1 were restricted to merged OTUs with the same taxonomic assignment. Results are represented as the mean \pm SEM. The Wilcoxon test was used to assess statistical significance of the bacterial composition between the different samples. Associations were considered to be significant after a false-discovery rate (FDR) correction of the $p$-value $(q<$ $0.05)$.

Beta diversity was assessed using weighted and unweighted UniFrac distances. The weighted Unifrac metric is weighted by the difference in the abundance of OTUs from each community, whereas unweighted Unifrac only considers the absence/presence of the OTUs providing different information. The link between the different groups of mice and bacterial microbial 
1 profiles was addressed by performing an ANOSIM test with 10,000 permutations on the beta 2 diversity metrics described above.

3 Functional composition of the intestinal metagenome was predicted using Phylogenetic Investigation of Communities by Reconstruction of Unobserved States (PICRUSt) ${ }^{9}$. This is a computational approach that accurately predicts the abundance of gene families in the microbiota and thus provides information about the functional composition of the microbial community. Linear discriminant analysis (LDA) effect size (LEfSe) analysis was performed to identify the taxa and functions displaying the largest differences in abundance in the microbiota between groups ${ }^{10}$. Only taxa and functions with an LDA score $>2$ and a significance of $<0.05$, as determined by Wilcoxon signed-rank tests, are shown. LEfSe and PICRUSt were accessed online (http://huttenhower.sph.harvard.edu/galaxy/).

Analysis of fecal metabolites by gas chromatography coupled to a triple quadrupole mass spectrometer. Fecal metabolites were measured using the GC-MS/MS method as previously described ${ }^{11}$. Briefly, about $20 \mathrm{mg}$ of biological material for each sample were first weighted and solubilized into microcentrifuge tubes with $500 \mu \mathrm{L}$ of MilliQ water $\left(+4^{\circ} \mathrm{C}\right)$. Samples were snap frozen in liquid nitrogen, then thaw at room temperature on the bench, while they were thoroughly vortex. Samples were splitted in two parts: the first $200 \mu 1$ were added to $300 \mu 1$ of cold methanol, the others $200 \mu \mathrm{l}$ were added to $300 \mu \mathrm{l}$ of cold TBME. The two aliquots were centrifugated 10 minutes at $15000 \mathrm{~g}\left(+4^{\circ} \mathrm{C}\right)$. Concerning the TBME extraction, the upper layer was transferred in vial for direct injection into Gas Chromatography coupled with Mass Spectrometry (GC/MS, WAX method). Concerning the methanol extraction, $400 \mu 1$ of the supernatant were transferred and evaporated in microcentrifuge tubes at $40^{\circ} \mathrm{C}$ in a pneumatically assisted concentrator (Techne DB3, Staffordshire, UK). On dried extract, $300 \mu 1$ of methanol were added then splitted in two aliquots: the first $150 \mu \mathrm{L}$ used for GC/MS (HP5MS method) experiment in vial injection, the others $150 \mu \mathrm{L}$ used for the Ultra High Pressure Liquid Chromatography coupled by Mass Spectrometry (UHPLC/MS) experimentations. Concerning the GC/MS (HP5MS) aliquots, the $150 \mu \mathrm{L}$ were evaporated and $50 \mu \mathrm{L}$ of methoxyamine (20 $\mathrm{mg} / \mathrm{mL}$ in pyridine) was added on dried extracts, and stored at room temperature in dark, during 16 hours. The day after, $80 \mu \mathrm{L}$ of MSTFA was added and final derivatization occurred at $40^{\circ} \mathrm{C}$ during 30 minutes. Samples were then directly injected into GC-MS. tubes at $40^{\circ} \mathrm{C}$ in a pneumatically assisted concentrator (Techne DB3, Staffordshire, UK). The LC-MS dried extracts were solubilized with $450 \mu \mathrm{L}$ of MilliQ water and aliquoted in 3 
microcentrifuge tubes $(100 \mu \mathrm{L})$ for each $\mathrm{LC}$ method and one microcentrifuge tube for backup. Aliquots for analysis were transferred in LC vials and injected into UHPLC/MS or kept at $80^{\circ} \mathrm{C}$ until injection.

The GC-MS/MS method was performed on a 7890B gas chromatography (Agilent Technologies, Waldbronn, Germany) coupled to a triple quadrupole 7000C (Agilent Technologies, Waldbronn, Germany) equipped with a High sensitivity electronic impact source (EI) operating in positive mode.

The front inlet temperature was $250^{\circ} \mathrm{C}$, the injection was performed in splitless mode. The transfer line and the ion-source temperature were $250^{\circ} \mathrm{C}$ and $230^{\circ} \mathrm{C}$, respectively. The septum purge flow was fixed at $3 \mathrm{~mL} / \mathrm{min}$, the purge flow to split vent operated at $80 \mathrm{~mL} / \mathrm{min}$ during 1 min and gas saver mode was set to $15 \mathrm{~mL} / \mathrm{min}$ after $5 \mathrm{~min}$.

The helium gas flowed through the column (J\&WScientificHP-5MS, 30m x $0.25 \mathrm{~mm}$, i.d. 0.25 $\mathrm{mm}$, d.f., Agilent Technologies Inc.) at $1 \mathrm{~mL} / \mathrm{min}$. Column temperature was held at $60^{\circ} \mathrm{C}$ for 1 min, then raised to $210^{\circ} \mathrm{C}\left(10^{\circ} \mathrm{C} / \mathrm{min}\right)$, followed by a step to $230^{\circ} \mathrm{C}\left(5^{\circ} \mathrm{C} / \mathrm{min}\right)$ and reached $325^{\circ} \mathrm{C}\left(15^{\circ} \mathrm{C} / \mathrm{min}\right)$, and be hold at this temperature for $5 \mathrm{~min}$.

The scan mode used was the MRM for biological samples. Peak detection and integration of the analytes were performed using the Agilent Mass Hunter quantitative software (B.07.01).

All the statistical analysis and pathway annotations for the metabolites were carried out using MetaboAnalyst web tool (www.metaboanalyst.ca) ${ }^{12}$. Data was normalized using log transformation and Pareto-scaling. For multi group analysis, one-way ANOVA was performed followed by post-hoc analyses using Tukey's HSD. For predicting variance in samples, Principal Component Analysis (PCA) was performed. The significant pathways involved in the pectin effect were also identified using MetaboAnalyst tool ${ }^{13}$.

Measurement of tryptophan metabolites in the feces and plasma of patients. Indole derivatives were quantified HPLC-coupled to high resolution mass spectrometry as previously described 14.

\section{REFERENCES}

1-Llopis M, et al. (2016) Gut 65(5):830-839.

2-Wrzosek L, et al. (2018) Sci Rep 8(1):6854.

3-Bertola A, et al. (2013) Nature protocols 8(3):627-637.

4-Tomas J, et al. (2013) FASEB J. 27(2):645-655.

5-Masella AP, et al. (2012) BMC Bioinformatics 13:31. 
1 6-Caporaso JG, et al. (2010) Nat Methods 7(5):335-336.

2 7-Edgar RC (2010) Bioinformatics 26(19):2460-2461.

3 8-DeSantis TZ, et al. (2006) Appl. Environ. Microbiol. 72(7):5069-5072.

4 9-Langille MG, et al. (2013) Nat. Biotechnol. 31(9):814-821.

5 10-Segata N, et al. (2011) Genome Biol 12(6):R60.

6 11-Pietrocola F, et al. (2017) Autophagy 13(12):2163-2170.

7 12-Chong J, et al. (2018) Nucleic Acids Res 46(W1):W486-W494.

8 13-Xia J, et al. (2016) Curr Protoc Bioinformatics 55:14 10 11-14 1091.

9 14-Lefevre A, et al. (2019) Talanta 195:593-598. 


\section{Supplementary Figure 1}

a

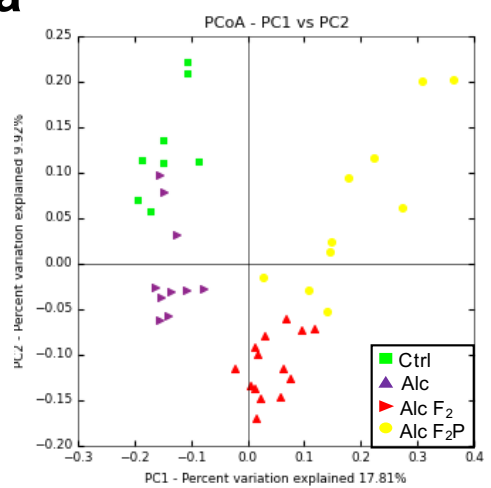

C

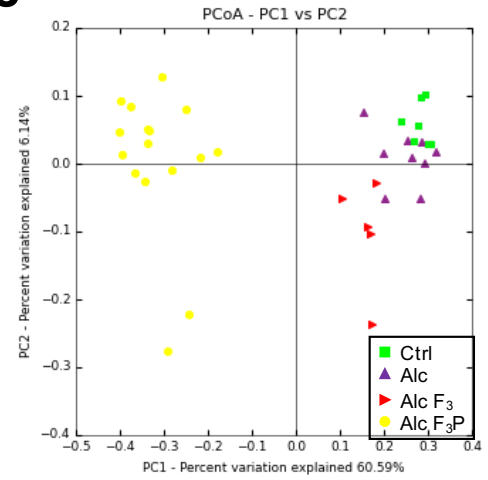

b

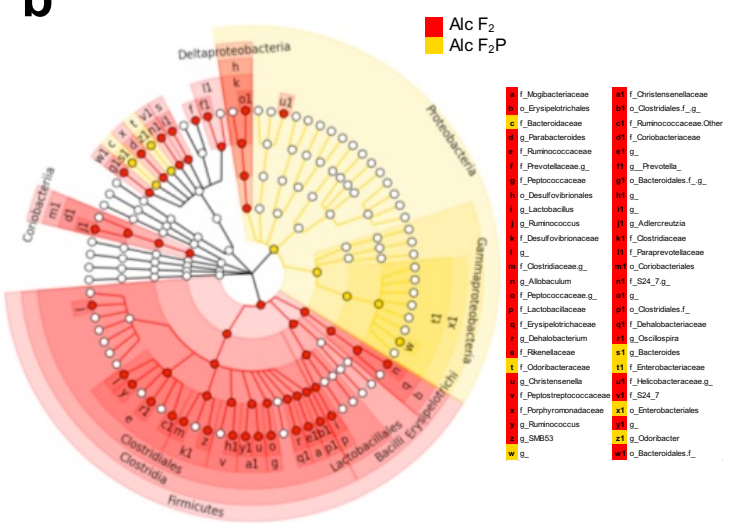

d

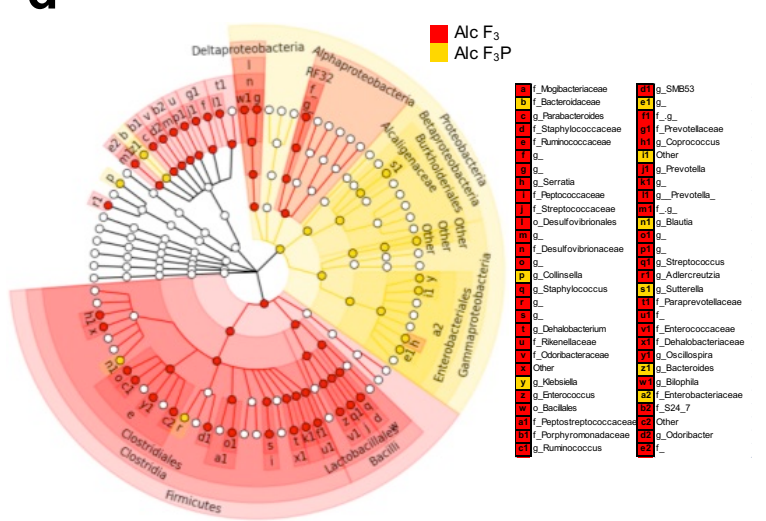

Supplementary Figure 1. The composition of the intestinal microbiota of mice humanized with feces from two patients with severe alcoholic hepatitis is modified by pectin treatment. Ctrl, control-fed mice; Alc, alcohol-fed mice; Alc F2 and Alc F3, alcohol-fed mice humanized with the microbiota from a patient with sAH (patient F2 or F3); Alc F2 P6.5 and Alc F3 P6.5, alcohol-fed mice humanized with the microbiota from a patient with sAH (patient F2 or F3) and treated with $6.5 \%$ pectin. (a) Principal Coordinate Analysis (PCoA) p lot showing the unweighted UniFrac distance ( $p<0.001, \mathrm{R}=0.59$, ANOSIM test, 10,000 permutations, using the first 5 PC). (b) Cladograms showing the taxa most differentially associated with Alc F2 (red) or Alc F2 P6.5 mice (yellow) (Wilcoxon rank-sum test). Circle sizes in the cladogram plot are proportional to bacterial abundance. The circles represent, going from the inner to outer circle: phyla, genus, class, order, and family. Mice per group for $\mathbf{a}$ and $\mathbf{b}$ : Ctrl $(\mathrm{n}=8)$, Alc $(\mathrm{n}=10)$, Alc F2 $(\mathrm{n}=15)$, and Alc F2 P6.5 $(\mathrm{n}=10)$. (c) PCoA plot showing the unweighted UniFrac distance $(\mathrm{p}<0.001$, $\mathrm{R}=0.68$, ANOSIM test, 10,000 permutations, using the first $5 \mathrm{PC}$ ). (d) Cladograms showing the taxa most differentially associated with Alc F3 (red) or Alc F3 P6.5 mice (yellow) (Wilcoxon rank-sum test). Mice per group in $\mathbf{c}$ and d: Ctrl (n=7), Alc (n=9), Alc F3 (n=5), Alc F3 P0.4 (n=8), Alc F3 P1 (n=11), Alc F3 P2 $(n=16)$, and Alc F3 P6.5 (n=16). 


\section{Supplementary Figure 2}

\section{1}

$2 \mathbf{a}$

\begin{tabular}{|c|c|c|c|}
\hline $\begin{array}{c}\text { Animal facility } \\
\text { adaptation }\end{array}$ & $\begin{array}{c}\text { Semi liquid diet } \\
\text { adaptation }\end{array}$ & Increase $\%$ \\
\hline & \\
\hline
\end{tabular}

b

C

d
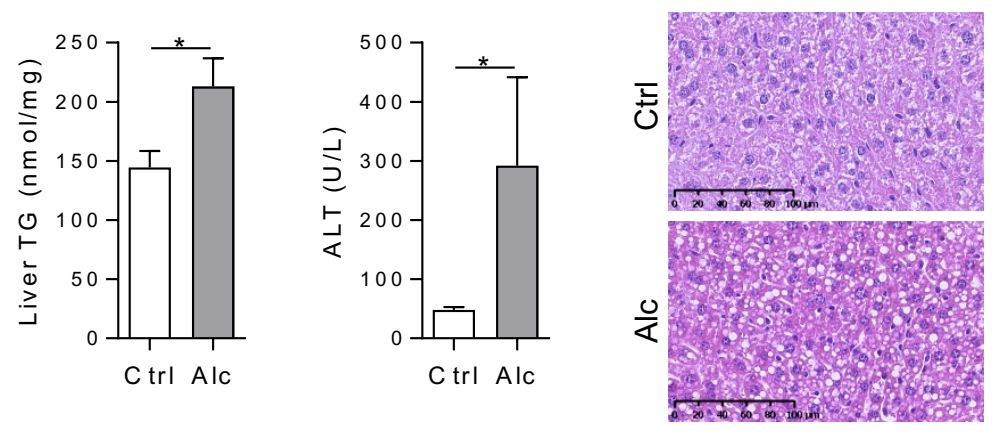

$\mathbf{e}$
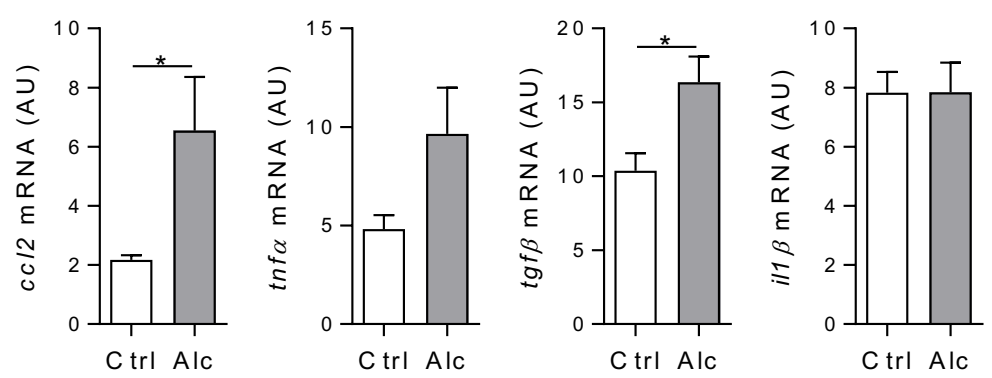

$\mathbf{f}$

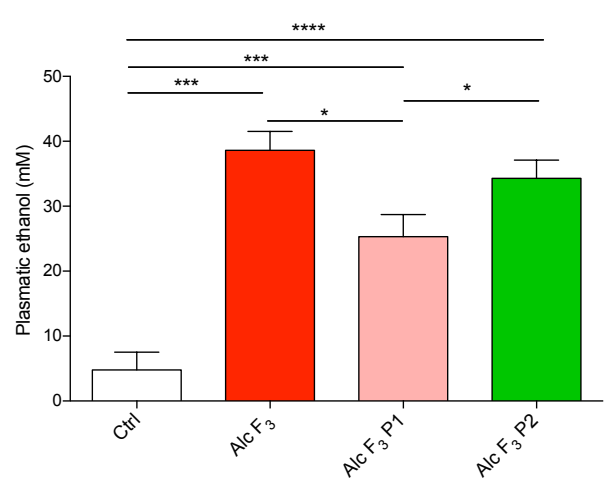

Supplementary Figure 2. Alcohol-induced liver injury after the alcohol adaptation period. Ctrl, control-fed mice ( $\mathrm{n}=11$ ); Alc, alcohol-fed mice $(\mathrm{n}=11)$. (a) Experimental design: mice were progressively adapted to a semi-liquid, Lieber DeCarli (LDC) diet, then to an ethanol diet, using increasing doses of ethanol (2-4\%) for seven days. (b) ALT level. (c) Liver triglyceride quantification. (d) Representative pictures of liver sections stained with haematoxylin-eosin in control and alcohol-fed mice showing steatosis, scale bar $100 \mu \mathrm{m}$. (e) Liver mRNA levels of inflammation markers were determined by qPCR: $c c l 2$, $\operatorname{tnf} \alpha$, tgf $\beta$, and $i l 1 \beta$, normalized to that of the gapdh gene. (f) Plasmatic alcohol in control mice (Ctrl) and alcohol fed mice transplanted with MI of sAH patient (Alc $\mathrm{sAH})$ and treated with $1 \%$ or $2 \%$ of pectin. Results are shown as the mean \pm SEM. Significant results for $* \mathrm{p}<0.05, * * * \mathrm{p}<0.0002$, $* * * * \mathrm{p}<0.0001$ were determined by the Mann-Whitney test. 


\section{Supplementary Figure 3}

a

b
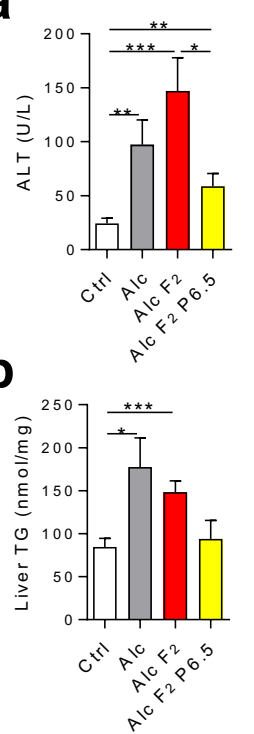

C
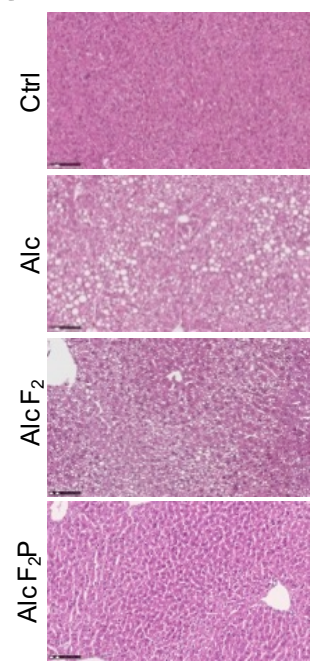

d
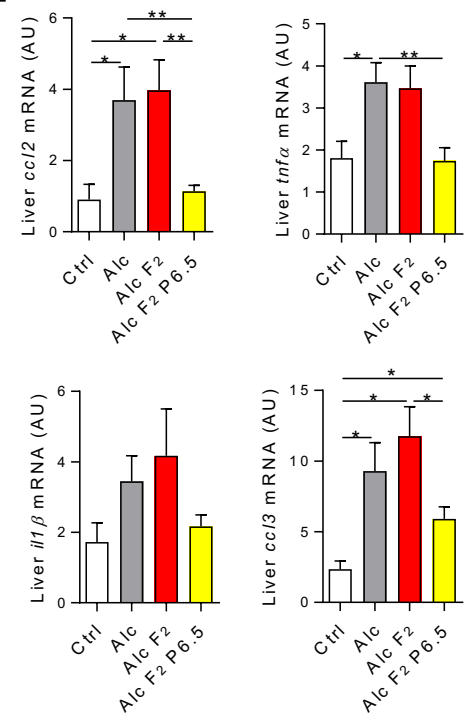

Supplementary Figure 3. Pectin treatment reverses liver lesions in mice humanized with the intestinal microbiota from a patient with severe alcoholic hepatitis. Ctrl, control-fed mice; Alc, alcohol-fed mice; Alc F2, alcohol-fed mice humanized with the microbiota from a patient with sAH (patient F2); Alc F2 P6.5, alcohol-fed mice humanized with the microbiota from a patient with sAH (patient F2) and treated with 6.5\% pectin. (a) ALT level in Ctrl (n=8), Alc (n=8), Alc F2 (n=16), and Alc F2 P6.5 $(n=10)$ mice. (b) Liver triglyceride quantification in Ctrl $(n=8)$, Alc $(n=8)$, Alc F2 (n=14), and Alc F2 P6.5 (n=8) mice. (c) Representative images of liver sections stained with haematoxylin-eosin, scale bar $100 \mu \mathrm{m}$. (d) Liver mRNA levels determined by qPCR: $c c l 2$, tnf $\alpha$, ill $\beta$ and $c c l 3$ normalized to that of the gapdh gene in Ctrl (n=4), Alc (n=5), Alc F2 (n=16), and Alc F2 P6.5 $(\mathrm{n}=9)$ mice. Results are shown as the mean \pm SEM. Significant results for $* \mathrm{p}<0.05, * * \mathrm{p}<0.01$, and $* * * \mathrm{p}<0.001$ were determined by Mann-Whitney tests unless stated otherwise. 


\section{Supplementary Figure 4}

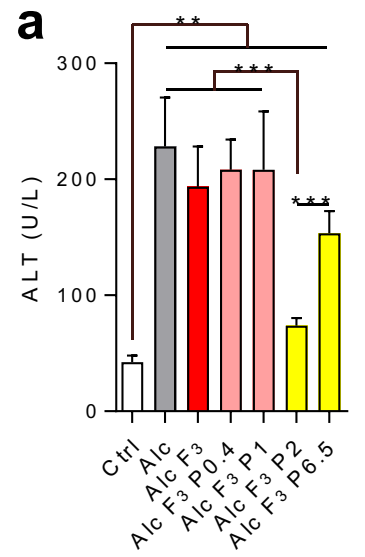

C

b
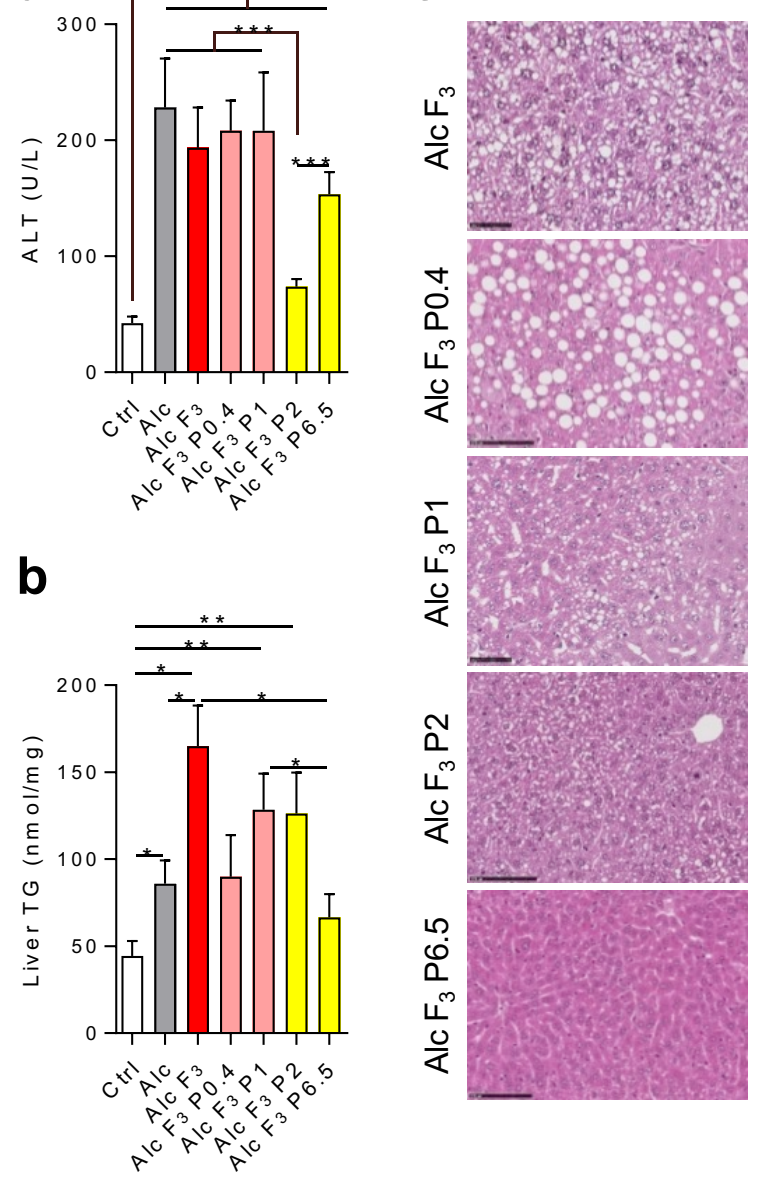

Supplementary Figure 4. Dose-dependent effect of pectin on liver and intestinal barrier function. Ctrl, control-fed mice; Alc, alcohol-fed mice; Alc F3, alcohol-fed mice humanized with the microbiota from a patient with s AH (patient F3); Alc F3 P0.4, Alc F3 P1, Alc F3 P2, and Alc F3 P6.5, alcohol-fed mice humanized with the microbiota from a patient with sAH (patient F3) and treated with 0.4, 1, 2, or 6.5\% pectin, respectively. (a) AL T levels in Ctrl ( $n=8)$, Alc ( $n=9)$, Alc F3 (n=6), Alc F3 P0.4 (n=9), Alc F3 P1 (n=12), Alc F3 P2 (n=15), and Alc F3 P6.5 (n=16) mice. (b) Liver triglyceride quantification in Ctrl (n=8), Alc (n=9), Alc F3 (n=3), Alc F3 P0.4 (n=7), Alc F3 P1 (n=10), Alc F3 P2 (n=16), and Alc F3 P6.5 ( $=14)$ mice. (c) Representative images of liver sections stained with ha ema toxylin-eosin, scale bar $400 \mu \mathrm{m}$. Results are shown as the mean \pm SEM. Sign ificant results for *p $<0.05, * * \mathrm{p}<0.01$, and $* * * \mathrm{p}<0.001$ were determined by Mann-Whitney tests unless stated otherwise. 


\section{Supplementary Figure 5}

a

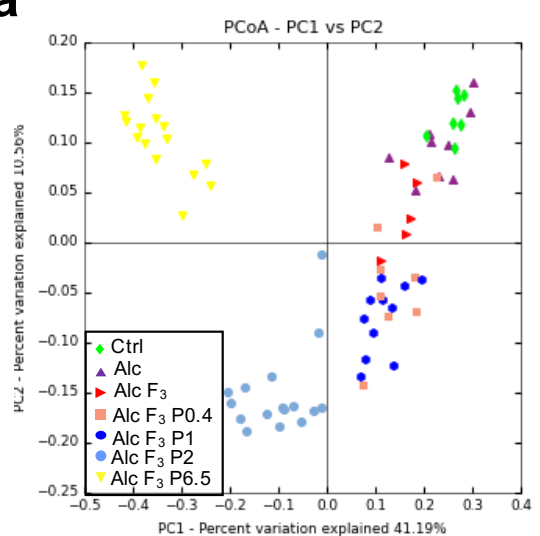

b

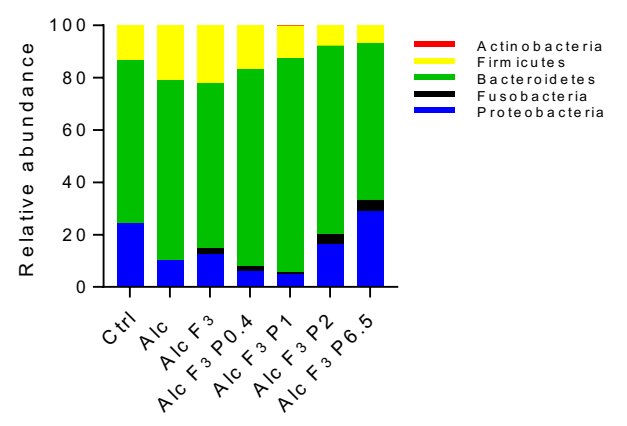

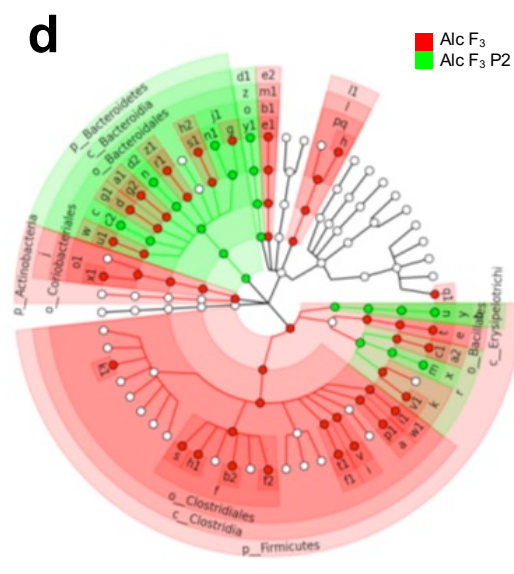

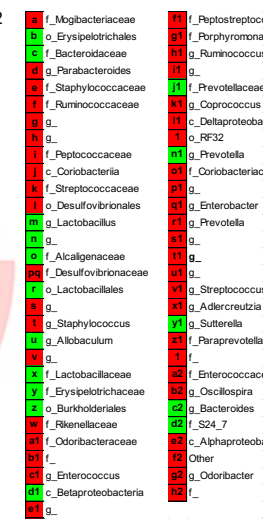

C

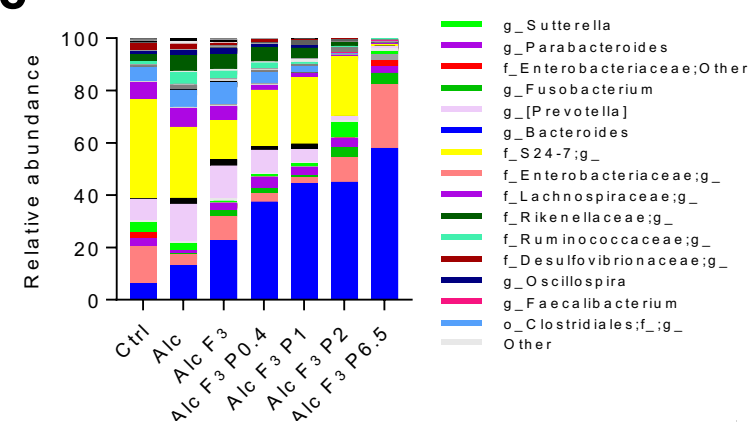

e

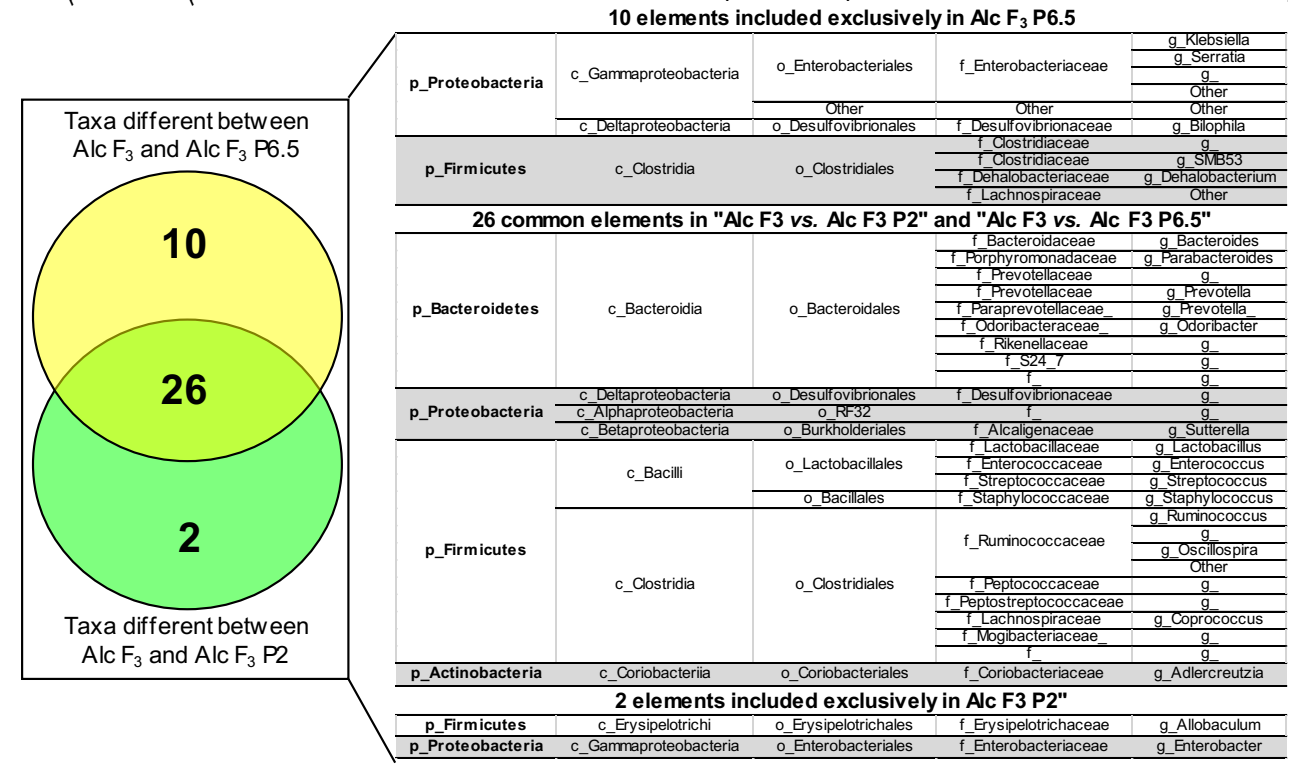

Supplementary Figure 5. Gut microbiota composition of mice humanized with intestinal microbiota from a patient with severe alcoholic and treated with different doses of pectin. Alc, alcohol-fed mice $(n=5)$; Ctrl, control-fed mice $(n=7)$; Alc $\mathrm{F}_{3}(\mathrm{n}=5)$, alcohol-fed mice humanized with the microbiota from a patient with $\mathrm{sAH}$ (patient $\left.\mathrm{F}_{3}\right) ; \mathrm{Alc}_{3} \mathrm{P} 0.4(\mathrm{n}=8)$, Alc $\mathrm{F}_{3} \mathrm{P} 1$ $(n=11), A_{1} F_{3}$ P2 $(n=16)$, Alc $F_{3}$ P6.5 $(n=16)$, alcohol-fed mice humanized with the microbiota from a patient with sAH (patient $\mathrm{F}_{3}$ ) and treated with pectin $0.4 \%, 1 \%, 2 \%$ or $6.5 \%$ respectively. (a) PCoA plot showing the unweighted UniFrac distance (p < $0.001, \mathrm{R}=0.75$, ANOSIM test, 10,000 permutations, using the first $5 \mathrm{PC}$ ). (b) Bacterial taxon-based analysis at the phylum and (c) genus level in faecal microbiota (d) LDA effect size (LEfSe) cladograms showing the taxa most differentially associated with Alc $\mathrm{F}_{3}$ (red) or $\mathrm{Alc}_{3} \mathrm{P} 2$ mice (green) (Wilcoxon rank-sum test). Circle sizes in the cladogram plot are proportional to bacterial abundance. The circles represent, going from the inner circ le to the outer circle: phyla, genus, class, order, and family. (e) Venn diagram based on the taxa different in LEfSe analysis between Alc $F_{3}$ vs. Alc $F_{3}$ P6.5 and Alc $F_{3}$ vs. Alc $F_{3}$ P2 and the corresponding common taxa between these two comparisons. 


\section{Supplementary Figure 6}

a
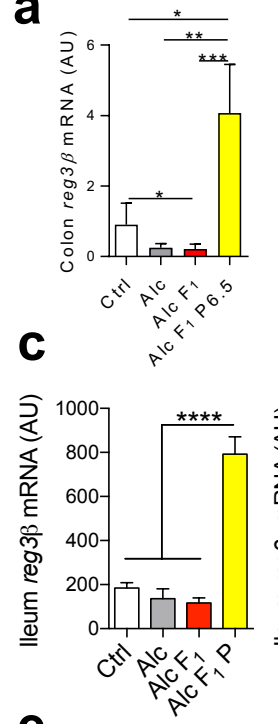

e

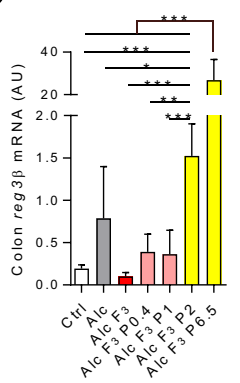

h

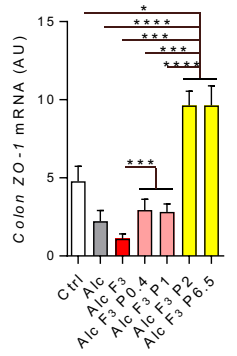

b
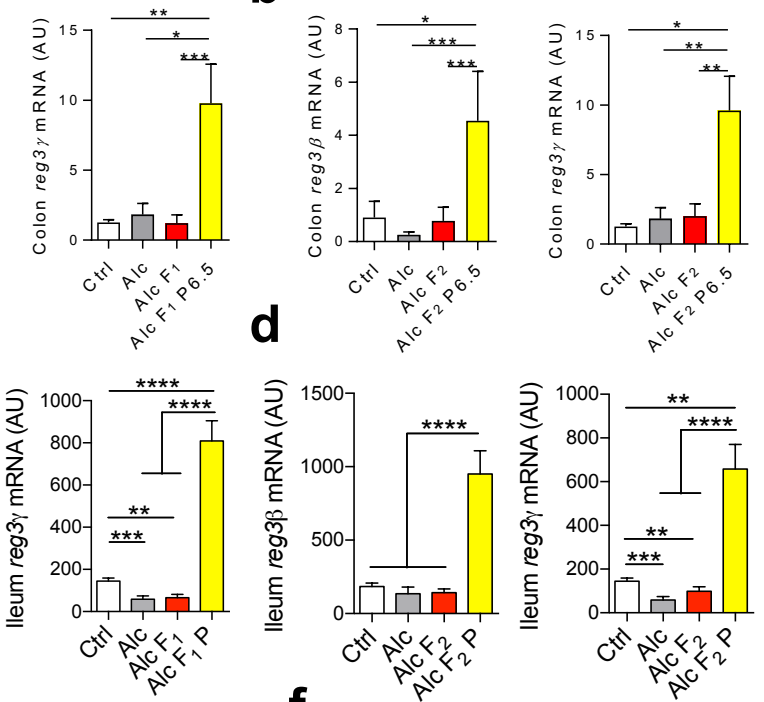

$\mathbf{f}$
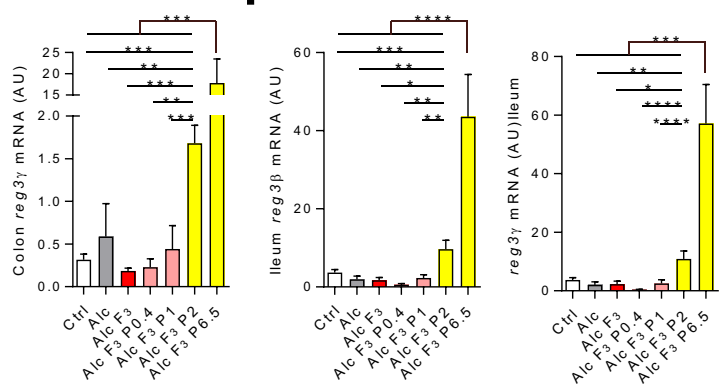

g

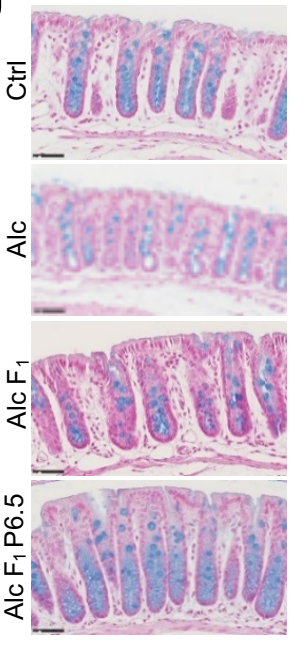

i
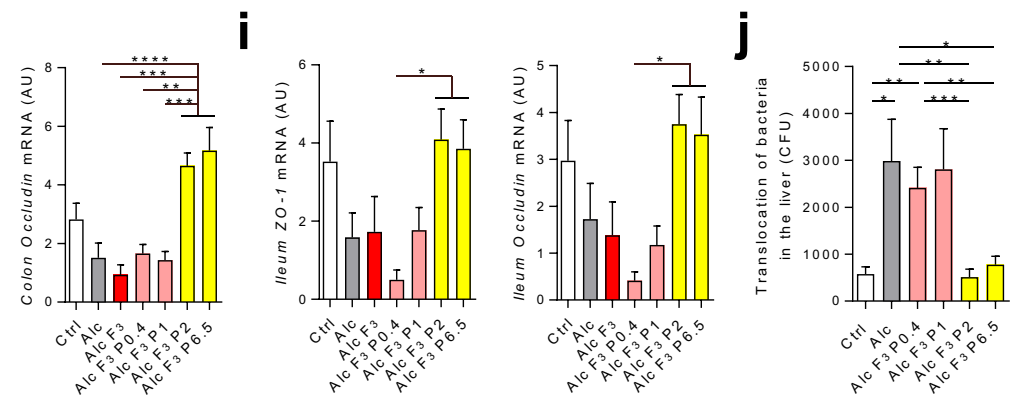

Supplementary Figure 6. Pectin treatment improves intestinal barrier function. Ctrl, control-fed mice; Alc, alcohol-fed mice; Alc F1, Alc F2 and Alc F3, alcohol-fed mice humanized with the microbiota from a patient with sAH (patients F1, F2 or F3); Alc F1 P6.5, Alc F2 P6.5 and Alc F3 P6.5, alcohol-fed mice humanized with the microbiota from a patient with sAH (patient 1, 2, or 3 ) and treated with $6.5 \%$ pectin. (a-f) Colon and ileum mRNA levels determined by qPCR: reg $3 \beta$ and reg $3 \gamma$ normalized to that of the gapdh gene. (e) Representative images of colon sections stained with Alcian blue, scale bar $50 \mu \mathrm{m}$. (h) Colon and (i) ileum mRNA levels determined by qPCR: $Z O-1$ and occludin normalized to that of the 18 sgene. (j) Culture of bacteria in the liver. For a$\mathrm{d}$ and g: Ctrl $(\mathrm{n}=8)$, Alc $(\mathrm{n}=8)$, Alc F1 $(\mathrm{n}=12)$, Alc F1 P6.5 $(\mathrm{n}=10)$, Alc F2 $(\mathrm{n}=16)$ and Alc F2 P6.5 (n=10) mice. For e-j: Ctrl $(\mathrm{n}=8)$, Alc $(\mathrm{n}=9)$, Alc F3 P0.4 (n=9), Alc F3 P1 ( $=12)$, Alc F3 P2 (n=13), and Alc F3 P6.5 ( $\mathrm{n}=13)$ mice. Results are shown as the mean \pm SEM. Significant results for ${ }^{*} \mathrm{p}<0.05,{ }^{* *} \mathrm{p}<0.01$, and ${ }^{* * *} \mathrm{p}<0.001$ were determined by Mann-Whitney tests unless stated otherwise. 


\section{Supplementary Figure 7}
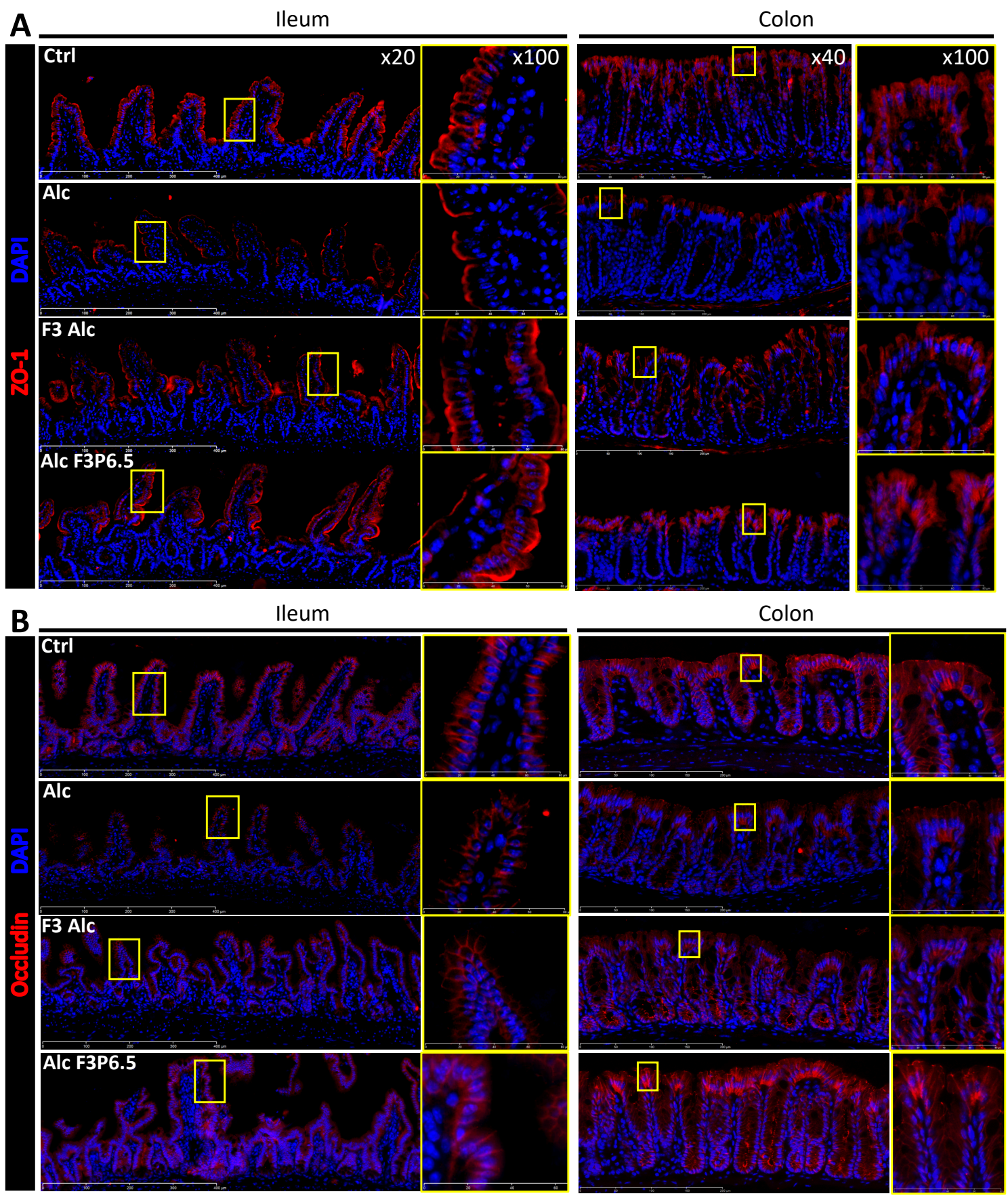

Supplementary Figure 7. Representative panels for the expression of tight junction proteins. (A) ZO-1 and (B) occludin expression in the ileum and colon. 


\section{Supplementary Figure 8}

a

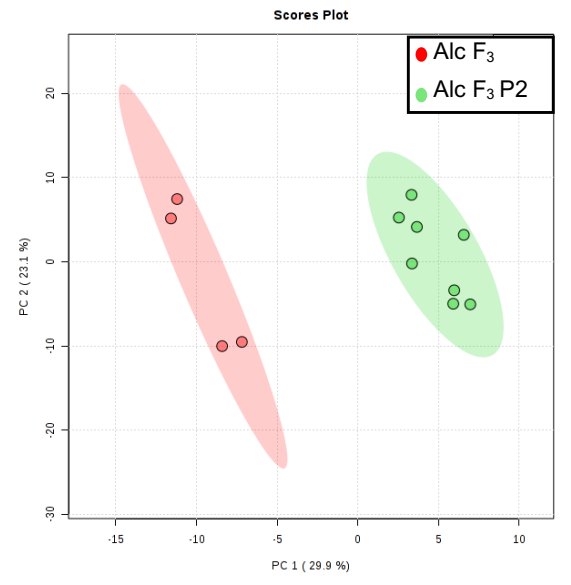

b

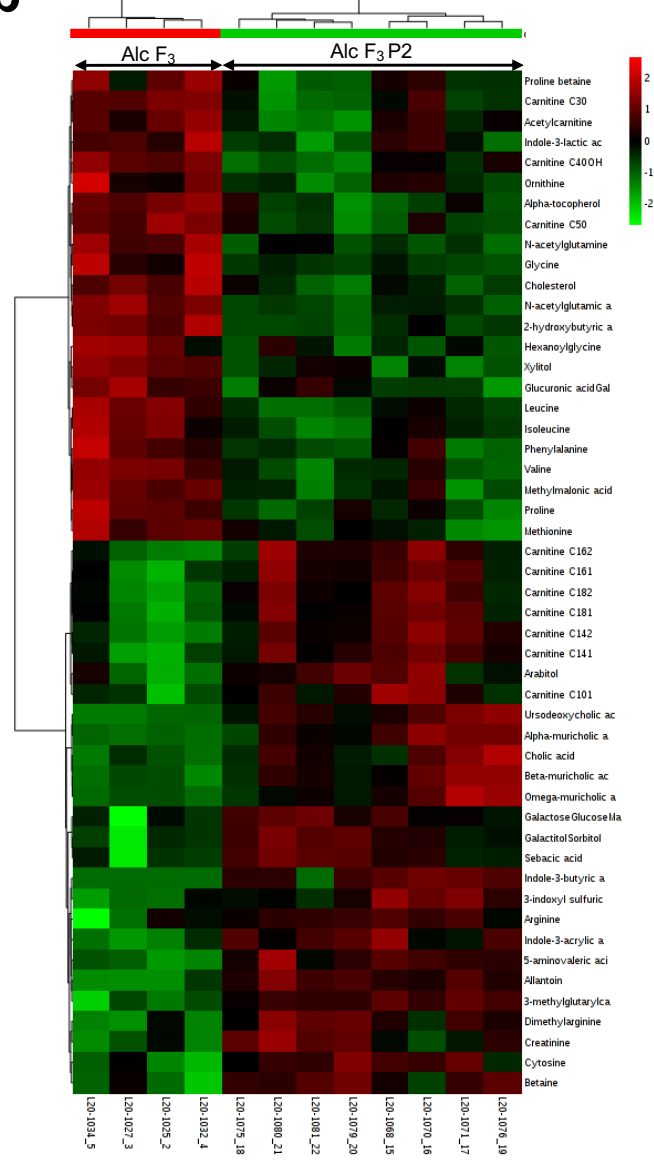

Supplementary Figure 8. The fecal metabolomic profile in mice humanized with intestinal microbiota from a patient with severe alcoholic hepatitis is modified by pectin. Alc $\mathrm{F} 3(\mathrm{n}=4)$, alcohol-fed mice humanized with the microbiota from a patient with sAH (patient F3), Alc F3 P2 (n=8), alcohol-fed mice humanized with the microbiota from a patient with sAH (patient F3) and treated with $2 \%$ pectin. (a) PCA ordination plot of all fecal metabolomic data. (b) Heatmap showing the first 60 metabolites ranked by t-tests between Alc F3 (red) and Alc F3 P2 (green). 
1 Supplementary table 1: Clinical characteristics of donor patients with severe alcoholic 2 hepatitis.

\begin{tabular}{cccc}
\hline Patient & $\mathbf{F}_{\mathbf{1}}$ & $\mathbf{F}_{\mathbf{2}}$ & $\mathbf{F}_{\mathbf{3}}$ \\
\hline Age $(\mathrm{yr})$ & 35 & 52 & 41 \\
Alcohol consumption $(\mathrm{g} / \mathrm{d})$ & 120 & 160 & 90 \\
Duration of alcohol intake $(\mathrm{yr})$ & 16 & 20 & 18 \\
BMI $(\mathrm{kg} / \mathrm{m} 2)$ & 29 & 21 & 23 \\
AST $(\mathrm{IU} / \mathrm{L})$ & 193 & 153 & 434 \\
Albumin $(\mathrm{g} / \mathrm{L})$ & 23 & 27 & 21 \\
Blood glucose (mmol/L) & & 5 & 5.6 \\
Triglycerides (g/L) & & 0.81 & 1.37 \\
PT (\%) & 25 & 30 & 26 \\
Bilirubin $(\mu \mathrm{mol} / \mathrm{L})$ & 569 & 92 & 220 \\
Maddrey discriminant function & 101 & 64 & 80 \\
MELD & 34 & 25 & 26 \\
\hline
\end{tabular}

BMI: body mass index; AST: aspartate aminotransferase; PT: prothrombin time as percentage of control

3

4 
Supplementary table 2: Predicted metabolomic pathways changes in intestinal microbiota

\begin{tabular}{|c|c|c|c|c|c|c|c|c|c|c|}
\hline \multirow{2}{*}{$\begin{array}{c}\text { Picrust predicted metabolic } \\
\text { pathways }\end{array}$} & \multirow[b]{2}{*}{ Pathway } & \multicolumn{3}{|c|}{ Alc F1 vs Alc F1 P6.5 } & \multicolumn{3}{|c|}{$\begin{array}{c}\text { Alc F2 vs Alc F2 } \\
\text { P6.5 }\end{array}$} & \multicolumn{3}{|c|}{$\begin{array}{c}\text { Alc F3 vs Alc F3 } \\
\text { P6.5 }\end{array}$} \\
\hline & & $\begin{array}{c}\text { Increased } \\
\text { in }\end{array}$ & LDA & $\mathbf{p}$ & $\begin{array}{c}\text { Increased } \\
\text { in }\end{array}$ & LDA & $\mathbf{p}$ & $\begin{array}{c}\text { Increased } \\
\text { in }\end{array}$ & LDA & $\mathbf{p}$ \\
\hline \multirow{9}{*}{ 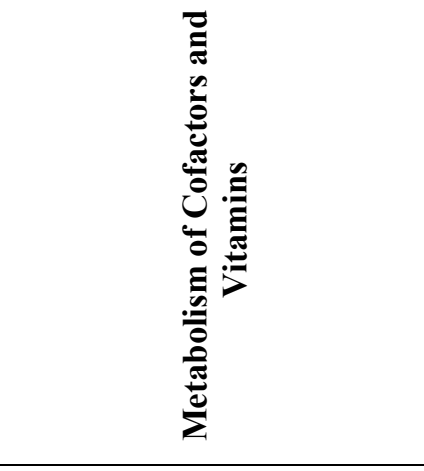 } & One carbon pool by folate & \multirow[t]{2}{*}{ AlcF1 } & \multirow[t]{2}{*}{2.55} & \multirow[t]{2}{*}{0.00} & \multirow[t]{2}{*}{ AlcF2 } & \multirow[t]{2}{*}{2.48} & \multirow[t]{2}{*}{0.00} & AlcF3 & 2.78 & 0.00 \\
\hline & Metabolism of cofactors and vitamins & & & & & & & AlcF3P6.5 & 2.41 & 0.00 \\
\hline & Ubiquinone and other terpenoid_quinone biosynthesis & \multirow{6}{*}{$\begin{array}{l}\text { AlcF1P6.5 } \\
\text { AlcF1 }\end{array}$} & \multirow{6}{*}{$\begin{array}{l}2.35 \\
2.13\end{array}$} & \multirow{6}{*}{$\begin{array}{l}0.00 \\
0.04\end{array}$} & \multirow{6}{*}{$\begin{array}{c}\text { AlcF2P6.5 } \\
\text { AlcF2 } \\
\text { AlcF2P6.5 }\end{array}$} & \multirow{2}{*}{$\begin{array}{l}2.33 \\
2.08\end{array}$} & \multirow{2}{*}{$\begin{array}{l}0.00 \\
0.00\end{array}$} & AlcF3P6.5 & 2.52 & 0.00 \\
\hline & Thiamine metabolism & & & & & & & AlcF3 & 2.29 & 0.00 \\
\hline & Retinol metabolism & & & & & 2.06 & 0.00 & AlcF3P6.5 & 2.20 & 0.00 \\
\hline & Riboflavin metabolism & & & & & & & AlcF3P6.5 & 2.31 & 0.00 \\
\hline & Folate biosynthesis & & & & & & & AlcF3 & 2.09 & 0.05 \\
\hline & Nicotinate and nicotinamide metabolism & & & & & & & AlcF3 & 2.06 & 0.05 \\
\hline & Pantothenate and CoA biosynthesis & AlcF1 & 2.29 & 0.00 & AlcF2 & 2.37 & 0.00 & AlcF3 & 2.45 & 0.00 \\
\hline & Phenylalanine metabolism & AlcF1P6.5 & 2.19 & 0.00 & AlcF2P6.5 & 2.32 & 0.00 & AlcF3P6.5 & 2.66 & 0.00 \\
\hline & Lysine biosynthesis & AlcF1 & 2.56 & 0.00 & AlcF2 & 2.57 & 0.00 & AlcF3 & 2.69 & 0.00 \\
\hline & Valine_leucine and isoleucine biosynthesis & AlcF1P6.5 & 2.10 & 0.00 & AlcF2P6.5 & 2.33 & 0.00 & AlcF3 & 2.47 & 0.00 \\
\hline 登 & Valine_leucine and isoleucine degradation & AlcF1 & 2.28 & 0.01 & AlcF2 & 2.37 & 0.00 & AlcF3P6.5 & 2.20 & 0.00 \\
\hline$\frac{0}{0}$ & Phenylalanine_tyrosine and tryptophan biosynthesis & AlcF1 & 2.46 & 0.01 & AlcF2 & 2.39 & 0.00 & AlcF3 & 2.62 & 0.00 \\
\hline$\sum$ & Aminoacidrelated enzymes & AlcF1 & 2.79 & 0.00 & AlcF2 & 2.76 & 0.00 & AlcF3 & 2.98 & 0.00 \\
\hline 产 & Lysine degradation & AlcF1P6.5 & 2.31 & 0.01 & AlcF2P6.5 & 2.21 & 0.00 & AlcF3P6.5 & 2.37 & 0.01 \\
\hline$\stackrel{\varrho}{=}$ & Tyrosine metabolism & AlcF1P6.5 & 2.07 & 0.01 & AlcF2P6.5 & 2.11 & 0.00 & AlcF3P6.5 & 2.28 & 0.01 \\
\hline 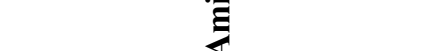 & Cysteine and methionine metabolism & & & & AlcF2 & 2.20 & 0.00 & AlcF3 & 2.20 & 0.00 \\
\hline & Histidine metabolism & & & & & & & AlcF3 & 2.70 & 0.00 \\
\hline & Tryptophan metabolism & & & & & & & AlcF3P6.5 & 2.18 & 0.05 \\
\hline & Phenylpropanoid biosynthesis & & & & & & & AlcF3 & 2.43 & 0.00 \\
\hline & Glutathione metabolism & AlcF1P6.5 & 2.42 & 0.00 & AlcF2P6.5 & 2.48 & 0.00 & AlcF3P6.5 & 2.61 & 0.00 \\
\hline Other Amino Acids & Cyanoaminoacid metabolism & AlcF1P6.5 & 2.12 & 0.02 & & & & AlcF3 & 2.46 & 0.00 \\
\hline & D_Alanine metabolism & & & & & & & AlcF3 & 2.07 & 0.00 \\
\hline & Methane metabolism & AlcF1 & 2.64 & 0.01 & AlcF2 & 2.62 & 0.00 & AlcF3 & 2.76 & 0.00 \\
\hline & Sulfur metabolism & AlcF1P6.5 & 2.03 & 0.00 & AlcF2P6.5 & 2.06 & 0.00 & AlcF3P6.5 & 2.36 & 0.00 \\
\hline
\end{tabular}




\begin{tabular}{|c|c|c|c|c|c|c|c|c|c|c|}
\hline & Nitrogen metabolism & AlcF1P6.5 & 2.49 & 0.00 & AlcF2P6.5 & 2.66 & 0.00 & AlcF3P6.5 & 2.84 & 0.00 \\
\hline & Photosynthesis proteins & AlcF1 & 2.28 & 0.00 & AlcF2 & 2.16 & 0.00 & AlcF3 & 2.45 & 0.00 \\
\hline & Photosynthesis & AlcF1 & 2.29 & 0.00 & AlcF2 & 2.16 & 0.00 & AlcF3 & 2.46 & 0.00 \\
\hline & Oxidative phosphorylation & & & & & & & AlcF3 & 2.67 & 0.01 \\
\hline \multirow{7}{*}{ 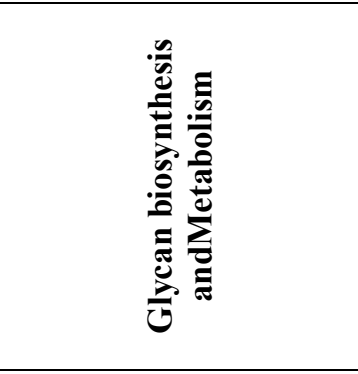 } & Glycosyl transferases & AlcF1P6.5 & 2.31 & 0.01 & AlcF2P6.5 & 2.42 & 0.00 & AlcF3P6.5 & 2.52 & 0.00 \\
\hline & Lipopolysaccharide biosynthesis proteins & AlcF1P6.5 & 2.42 & 0.03 & AlcF2P6.5 & 2.50 & 0.01 & AlcF3P6.5 & 2.81 & 0.00 \\
\hline & Peptidoglycan biosynthesis & AlcF1 & 2.60 & 0.00 & AlcF2 & 2.60 & 0.00 & AlcF3 & 2.83 & 0.00 \\
\hline & Glycosaminoglyc and degradation & AlcF1P6.5 & 2.29 & 0.03 & AlcF2P6.5 & 2.55 & 0.00 & & & \\
\hline & Otherglyc and degradation & & & & AlcF2P6.5 & 2.87 & 0.00 & & & \\
\hline & Glycosphingolipid biosynthesis_ganglioseries & & & & AlcF2P6.5 & 2.35 & 0.00 & & & \\
\hline & Lipopolysaccharide biosynthesis & & & & AlcF2P6.5 & 2.28 & 0.01 & & & \\
\hline \multirow{3}{*}{$\begin{array}{l}\text { Biosynthesis of Other } \\
\text { Secondary Metabolites }\end{array}$} & Penicillin andcephalosporin biosynthesis & & & & AlcF2P6.5 & 2.03 & 0.00 & AlcF3P6.5 & 2.08 & 0.00 \\
\hline & Biosynthesis andbio degradation of secondary metabolites & AlcF1P6.5 & 2.20 & 0.00 & & & & & & \\
\hline & Butirosin and neomycin biosynthesis & & & & & & & AlcF3 & 2.22 & 0.00 \\
\hline \multirow{2}{*}{ Enzyme Families } & Proteinkinases & AlcF1P6.5 & 2.27 & 0.05 & & & & AlcF3P6.5 & 2.50 & 0.00 \\
\hline & Peptidases & AlcF1 & 2.68 & 0.00 & AlcF2 & 2.51 & 0.01 & AlcF3 & 2.79 & 0.00 \\
\hline \multirow{15}{*}{ 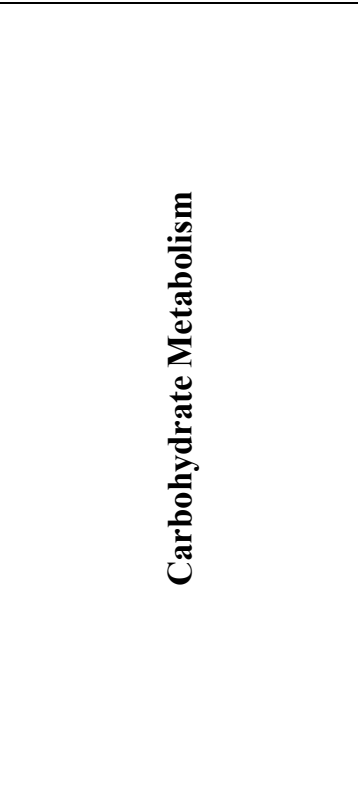 } & Galactose metabolism & AlcF1P6.5 & 2.65 & 0.00 & AlcF2P6.5 & 2.78 & 0.00 & AlcF3P6.5 & 2.53 & 0.05 \\
\hline & Glyoxylate and dicarboxylate metabolism & AlcF1P6.5 & 2.35 & 0.00 & AlcF2P6.5 & 2.45 & 0.00 & AlcF3P6.5 & 2.80 & 0.00 \\
\hline & Fructose and mannose metabolism & AlcF1P6.5 & 2.40 & 0.02 & AlcF2P6.5 & 2.63 & 0.00 & AlcF3P6.5 & 2.85 & 0.00 \\
\hline & Starch and sucrose metabolism & AlcF1P6.5 & 2.66 & 0.00 & AlcF2P6.5 & 2.41 & 0.00 & AlcF3 & 2.40 & 0.01 \\
\hline & Pentose and glucuronate interconversions & AlcF1P6.5 & 2.70 & 0.00 & AlcF2P6.5 & 2.79 & 0.00 & AlcF3P6.5 & 2.96 & 0.00 \\
\hline & Ascorbate and aldarate metabolism & AlcF1P6.5 & 2.29 & 0.02 & AlcF2P6.5 & 2.03 & 0.00 & AlcF3P6.5 & 2.51 & 0.01 \\
\hline & Pyruvate metabolism & & & & AlcF2P6.5 & 2.12 & 0.01 & AlcF3P6.5 & 2.51 & 0.00 \\
\hline & Propanoate metabolism & & & & AlcF2P6.5 & 2.23 & 0.04 & AlcF3P6.5 & 2.60 & 0.00 \\
\hline & Pentose phosphate pathway & & & & AlcF2P6.5 & 2.03 & 0.01 & AlcF3P6.5 & 2.41 & 0.00 \\
\hline & Glycolysis_Gluconeogenesis & & & & & & & AlcF3P6.5 & 2.02 & 0.01 \\
\hline & Butanoate metabolism & & & & & & & AlcF3P6.5 & 2.43 & 0.00 \\
\hline & Inositolphosphate metabolism & & & & & & & AlcF3P6.5 & 2.37 & 0.00 \\
\hline & Biosynthesis and biodegradation of secondary metabolites & & & & & & & AlcF3P6.5 & 2.38 & 0.00 \\
\hline & Carbohydrate metabolism & & & & & & & AlcF3P6.5 & 2.65 & 0.00 \\
\hline & Glycan biosynthesis and metabolism & & & & & & & AlcF3P6.5 & 2.11 & 0.01 \\
\hline
\end{tabular}




\begin{tabular}{|c|c|c|c|c|c|c|c|c|c|c|}
\hline & Aminosugar and nucleotidesugar metabolism & & & & AlcF2P6.5 & 2.51 & 0.00 & & & \\
\hline \multirow{7}{*}{ 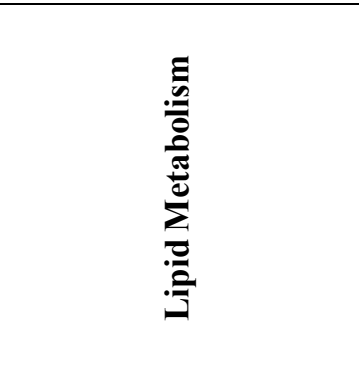 } & Fattyacid metabolism & AlcF1P6.5 & 2.29 & 0.03 & \multirow[t]{2}{*}{ AlcF2P6.5 } & \multirow[t]{2}{*}{2.33} & \multirow[t]{2}{*}{0.00} & \multirow{7}{*}{$\begin{array}{c}\text { AlcF3P6.5 } \\
\text { AlcF3P6.5 } \\
\text { AlcF3P6.5 } \\
\text { AlcF3 }\end{array}$} & \multirow{7}{*}{\multicolumn{2}{|c|}{$\begin{array}{ll}2.56 & 0.00 \\
2.37 & 0.00 \\
2.19 & 0.03 \\
2.29 & 0.00\end{array}$}} \\
\hline & Biosynthesis of unsaturated fatty acids & AlcF1P6.5 & 2.18 & 0.01 & & & & & & \\
\hline & Fattyacid biosynthesis & & & & AlcF2P6.5 & 2.02 & 0.00 & & & \\
\hline & Glycerophospholipid metabolism & & & & AlcF2 & 2.12 & 0.00 & & & \\
\hline & Glycerolipid metabolism & AlcF1 & 2.23 & 0.00 & AlcF2 & 2.31 & 0.00 & & & \\
\hline & Lipid biosynthesis proteins & & & & AlcF2P6.5 & 2.03 & 0.00 & & & \\
\hline & Sphingolipid metabolism & AlcF1P6.5 & 2.36 & 0.04 & AlcF2P6.5 & 2.58 & 0.00 & & & \\
\hline \multirow{9}{*}{ 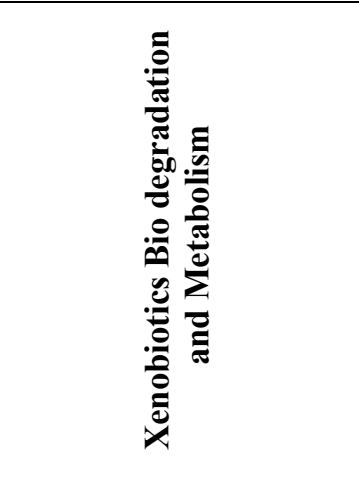 } & Drug metabolism_other enzymes & AlcF1 & 2.07 & 0.02 & AlcF2 & 2.05 & 0.01 & AlcF3 & 2.45 & 0.00 \\
\hline & Drug metabolism_cytochrome P450 & AlcF1P6.5 & 2.01 & 0.00 & AlcF2P6.5 & 2.10 & 0.00 & AlcF3P6.5 & 2.21 & 0.00 \\
\hline & Metabolism of xenobiotics by cytochrome $\mathrm{P} 450$ & AlcF1P6.5 & 2.02 & 0.00 & AlcF2P6.5 & 2.08 & 0.00 & AlcF3P6.5 & 2.21 & 0.00 \\
\hline & Benzoate degradation & & & & AlcF2P6.5 & 2.06 & 0.04 & AlcF3P6.5 & 2.50 & 0.00 \\
\hline & Caprolactam degradation & AlcF1P6.5 & 2.07 & 0.04 & & & & AlcF3P6.5 & 2.27 & 0.00 \\
\hline & Naphthalene degradation & & & & AlcF2P6.5 & 2.03 & 0.00 & & & \\
\hline & Dioxin degradation & & & & & & & AlcF3P6.5 & 2.28 & 0.00 \\
\hline & Xylene degradation & & & & & & & AlcF3P6.5 & 2.20 & 0.00 \\
\hline & Toluene degradation & & & & & & & AlcF3P6.5 & 2.25 & 0.00 \\
\hline \multirow{2}{*}{ Nucleotide Metabolism } & Pyrimidine metabolism & AlcF1 & 2.98 & 0.00 & AlcF2 & 2.93 & 0.00 & AlcF3 & 3.16 & 0.00 \\
\hline & Purine metabolism & AlcF1 & 2.78 & 0.00 & AlcF2 & 2.65 & 0.00 & AlcF3 & 2.85 & 0.01 \\
\hline \multirow{5}{*}{ 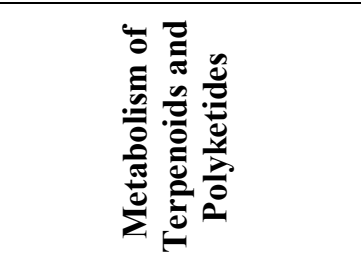 } & Prenyl transferases & AlcF1 & 2.20 & 0.01 & AlcF2 & 2.15 & 0.01 & AlcF3 & 2.43 & 0.00 \\
\hline & Terpenoid backbone biosynthesis & AlcF1 & 2.52 & 0.00 & AlcF2 & 2.44 & 0.00 & AlcF3 & 2.64 & 0.00 \\
\hline & Geraniol degradation & AlcF1P6.5 & 2.22 & 0.00 & AlcF2P6.5 & 2.24 & 0.00 & AlcF3P6.5 & 2.40 & 0.00 \\
\hline & Biosynthesis of siderophore group nonribosomal peptides & AlcF1P6.5 & 2.17 & 0.00 & AlcF2P6.5 & 2.02 & 0.00 & AlcF3P6.5 & 2.46 & 0.00 \\
\hline & Tetracycline biosynthesis & & & & & & & AlcF3P6.5 & 2.14 & 0.00 \\
\hline
\end{tabular}


Supplementary table 3: Pathways modified in pectin treated mice based on fecal metabolomic analysis

\begin{tabular}{|c|c|c|c|c|c|}
\hline Pathway Name & \begin{tabular}{|c|} 
Nb of \\
compounds \\
included in the \\
analysis
\end{tabular} & $\begin{array}{l}\text { Total } \\
\text { compounds } \\
\text { in pathway }\end{array}$ & $\mathbf{p}$ & FDR & Impact \\
\hline Inositol phosphate metabolism & 2 & 28 & 0.000 & 0.000 & 0.11163 \\
\hline Ascorbate and aldarate metabolism & 3 & 9 & 0.000 & 0.000 & 0.4 \\
\hline Starch and sucrose metabolism & 5 & 19 & 0.000 & 0.000 & 0.24448 \\
\hline Methane metabolism & 2 & 9 & 0.000 & 0.000 & 0.4 \\
\hline Cyanoamino acid metabolism & 2 & 6 & 0.000 & 0.000 & 0 \\
\hline Lysine biosynthesis & 1 & 4 & 0.000 & 0.000 & 0 \\
\hline Lysine degradation & 1 & 23 & 0.000 & 0.000 & 0 \\
\hline Biotin metabolism & 1 & 5 & 0.000 & 0.000 & 0 \\
\hline Purine metabolism & 13 & 68 & 0.000 & 0.000 & 0.14028 \\
\hline Tyrosine metabolism & 3 & 44 & 0.000 & 0.000 & 0.14045 \\
\hline Valine, leucine and isoleucine degradation & 5 & 38 & 0.000 & 0.000 & 0.0238 \\
\hline Porphyrin and chlorophyll metabolism & 2 & 27 & 0.000 & 0.000 & 0 \\
\hline Pentose and glucuronate interconversions & 5 & 16 & 0.000 & 0.000 & 0.26666 \\
\hline Valine, leucine and isoleucine biosynthesis & 6 & 11 & 0.000 & 0.000 & 0.99999 \\
\hline Histidine metabolism & 3 & 15 & 0.000 & 0.000 & 0.24194 \\
\hline Glyoxylate and dicarboxylate metabolism & 2 & 18 & 0.000 & 0.000 & 0.32258 \\
\hline Citrate cycle (TCA cycle) & 5 & 20 & 0.000 & 0.000 & 0.24593 \\
\hline D-Glutamine and D-glutamate metabolism & 3 & 5 & 0.000 & 0.000 & 1 \\
\hline Nitrogen metabolism & 4 & 9 & 0.000 & 0.000 & 0 \\
\hline Linoleic acid metabolism & 1 & 16 & 0.000 & 0.000 & 1 \\
\hline Pantothenate and CoA biosynthesis & 5 & 15 & 0.000 & 0.001 & 0.02041 \\
\hline Cysteine and methionine metabolism & 4 & 27 & 0.000 & 0.001 & 0.1351 \\
\hline Aminoacyl-tRNA biosynthesis & 18 & 69 & 0.000 & 0.001 & 0.12903 \\
\hline Glutathione metabolism & 6 & 26 & 0.000 & 0.001 & 0.09828 \\
\hline beta-Alanine metabolism & 4 & 17 & 0.000 & 0.001 & 0.44444 \\
\hline Butanoate metabolism & 6 & 22 & 0.000 & 0.001 & 0.02899 \\
\hline
\end{tabular}


Alanine, aspartate and glutamate metabolism Glycine, serine and threonine metabolism Ubiquinone and other terpenoid-quinone

biosynthesis

Primary bile acid biosynthesis

Sphingolipid metabolism

Phenylalanine, tyrosine and tryptophan

biosynthesis

Phenylalanine metabolism

Steroid biosynthesis

Steroid hormone biosynthesis

Pyrimidine metabolism

Galactose metabolism

Arginine and proline metabolism

Taurine and hypotaurine metabolism

Selenoamino acid metabolism

Pyruvate metabolism

Propanoate metabolism

Fatty acid elongation in mitochondria

Fatty acid metabolism

Glycerolipid metabolism

Pentose phosphate pathway

Glycolysis or Gluconeogenesis

Biosynthesis of unsaturated fatty acids

Nicotinate and nicotinamide metabolism

Fatty acid biosynthesis

Tryptophan metabolism

Glycerophospholipid metabolism

Amino sugar and nucleotide sugar metabolism Arachidonic acid metabolism

\begin{tabular}{|c|c|c|c|c|}
\hline 10 & 24 & 0.000 & 0.001 & 0.78269 \\
\hline 10 & 31 & 0.000 & 0.001 & 0.59903 \\
\hline 1 & 3 & 0.001 & 0.001 & 0 \\
\hline 5 & 46 & 0.001 & 0.001 & 0.12626 \\
\hline 2 & 21 & 0.001 & 0.001 & 0.01504 \\
\hline 2 & 4 & 0.001 & 0.002 & 1 \\
\hline 2 & 11 & 0.001 & 0.002 & 0.40741 \\
\hline 1 & 35 & 0.002 & 0.002 & 0.05394 \\
\hline 1 & 72 & 0.002 & 0.002 & 0.01689 \\
\hline 5 & 41 & 0.002 & 0.003 & 0.08292 \\
\hline 6 & 26 & 0.002 & 0.003 & 0.07627 \\
\hline 14 & 44 & 0.003 & 0.004 & 0.54477 \\
\hline 3 & 8 & 0.003 & 0.004 & 0.71428 \\
\hline 1 & 15 & 0.004 & 0.005 & 0 \\
\hline 2 & 23 & 0.004 & 0.006 & 0.18375 \\
\hline 2 & 20 & 0.006 & 0.007 & 0 \\
\hline 1 & 27 & 0.008 & 0.010 & 0 \\
\hline 1 & 39 & 0.008 & 0.010 & 0 \\
\hline 3 & 18 & 0.010 & 0.013 & 0.41129 \\
\hline 4 & 19 & 0.018 & 0.021 & 0.41291 \\
\hline 3 & 26 & 0.018 & 0.021 & 0.13406 \\
\hline 7 & 42 & 0.020 & 0.023 & 0 \\
\hline 2 & 13 & 0.034 & 0.038 & 0.2381 \\
\hline 4 & 43 & 0.035 & 0.038 & 0 \\
\hline 2 & 40 & 0.041 & 0.044 & 0.28702 \\
\hline 5 & 30 & 0.046 & 0.048 & 0.11297 \\
\hline 2 & 37 & 0.144 & 0.147 & 0.08988 \\
\hline 1 & 36 & 0.384 & 0.384 & 0.32601 \\
\hline
\end{tabular}

FDR: false discovery rate 
1 Supplementary table 4: Clinical characteristics of alcoholic patients for tryptophan pathway 2 analysis.

\begin{tabular}{lcc}
\hline & $\begin{array}{c}\text { Alcoholic patients } \\
\text { without alcoholic } \\
\text { hepatitis (noAH) } \\
(\mathrm{n}=15)\end{array}$ & $\begin{array}{c}\text { Alcoholic patients } \\
\text { with severe alcoholic } \\
\text { hepatitis (sAH) } \\
(\mathrm{n}=14)\end{array}$ \\
\hline Age (years) & $52.07 \pm 8.21$ & $55.79 \pm 12.4$ \\
Sex (male, $\%)$ & $10(67)$ & $13(93)$ \\
BMI (kg/m2) & $22.08 \pm 4.3$ & $25.56 \pm 5.05$ \\
Alcohol (g/day)* & $149.14 \pm 101.81$ & $77.86 \pm 36.2$ \\
Alcohol duration & $15.04 \pm 12.31$ & $21.23 \pm 10.37$ \\
years) & $11(73)$ & $9(64)$ \\
Smoking (yes, $\%)$ & $38.93 \pm 20.83$ & $131.36 \pm 105.03$ \\
AST (IU/L)** & $36.27 \pm 16.18$ & $42.5 \pm 16.58$ \\
ALT (IU/L) & $13.13 \pm 7.72$ & $229 \pm 221.84$ \\
Bilirubin** & $121.73 \pm 97.53$ & $423.43 \pm 384.51$ \\
GGT (IU/L)* & $211.93 \pm 72.48$ & $127 \pm 114.12$ \\
Platelets $\left(\times 10^{\wedge}\right.$ 9/L) $*$ & $98.67 \pm 4.13$ & $39 \pm 14.46$ \\
PT $(\%) * * *$ & $2.78 \pm 2.92$ & $22.84 \pm 7.5$ \\
MELD score*** & & \\
\hline BMI: body mass index, AST: aspartate transaminase, ALT: alanine transaminase, GGT: \\
gamma-glutamyltransferase, PT: prothrombin time, MELD: Model for End-Stage Liver \\
Disease. $*<0.05, * *<0.01, * * *<0.001$. Data are presented as mean \pm SD. \\
\hline
\end{tabular}


1 Supplementary table 5: Primer sequences used for q-PCR reactions

\begin{tabular}{|c|c|c|}
\hline Name & 5'- Forward - 3' & 5'-Reverse - 3' \\
\hline $18 \mathrm{~s}$ & GTAACCCGTTGAACCCCATT & CCATCCAATCGGTAGTAGCG \\
\hline ahrr & CATACGCCGC & GGTCCAGCTCTC \\
\hline ccl2 & GGTCCCTGTCATGCTTCTG & TCTGGACCCATTCCTTCTTG \\
\hline ccl3 & \multicolumn{2}{|c|}{ purchased from Qiagen, ref QT00248199 } \\
\hline cyp1a1 & CAGGATGTGTCTGGTTACTTTGAC & CTGGGCTACACAAGACTCTGTCTC \\
\hline gapdh & GTGGACCTCAT & TGTGAGGGAGATGCTCAGTG \\
\hline ill $\beta$ & AAGGTCCACGGGA & AGCTTCAGGCAGGCAGTATC \\
\hline il17 & ГТTAACTCCCTTGGCGCAAAA & CTTTCCCTCCGCATTGACAC \\
\hline il22 & ATGAGTTTTTCCC & GCTGGAAGTTGGACACCTCAA \\
\hline $\operatorname{reg} 3 \beta$ & GGCAACTTCACCTCACAT & TGGGAATGGAGTAACAATG \\
\hline $\operatorname{reg} 3 \gamma$ & CAAGATGTCCTGAGGGC & CCATCTTCACGTAGCAGC \\
\hline scd1 & CCGGAGACCCTTAGATCGA & TAGCCTGTAAAAGATTTCTGCAAA \\
\hline $\operatorname{tg} f \beta$ & GCAACATGTGGAACTCTACCAGAA & GACGTCAAAAGACAGCCACTCA \\
\hline $\operatorname{tnfa}$ & TGGGAGTAGACAAGGTACAACCC & CATCTTCTCAAAATTCGAGTGACAA \\
\hline
\end{tabular}

